\title{
Freeze-in dark matter from secret neutrino interactions
}

\author{
Yong Du, ${ }^{a, b}$ Fei Huang, ${ }^{a, c}$ Hao-Lin $\mathbf{L i}^{a}$ and Jiang-Hao $\mathbf{Y u}^{a, d, e, f, 1}$ \\ ${ }^{a}$ CAS Key Laboratory of Theoretical Physics, Institute of Theoretical Physics, \\ Chinese Academy of Sciences, Beijing 100190, China \\ ${ }^{b}$ Amherst Center for Fundamental Interactions, Physics Department, \\ University of Massachusetts Amherst, Amherst, MA 01003, U.S.A. \\ ${ }^{c}$ Department of Physics and Astronomy, University of California, \\ Irvine, CA 92697, U.S.A. \\ ${ }^{d}$ School of Physical Sciences, University of Chinese Academy of Sciences, \\ Beijing 100049, China \\ ${ }^{e}$ School of Fundamental Physics and Mathematical Sciences, \\ Hangzhou Institute for Advanced Study, University of Chinese Academy of Sciences, \\ Hangzhou 310024, China \\ ${ }^{f}$ International Centre for Theoretical Physics Asia-Pacific, \\ Beijing/Hangzhou, China \\ E-mail: yongdu@umass.edu, huangf4@uci.edu, lihaolin@itp.ac.cn, \\ jhyu@itp.ac.cn
}

ABSTRACT: We investigate a simplified freeze-in dark-matter model in which the dark matter only interacts with the standard-model neutrinos via a light scalar. The extremely small coupling for the freeze-in mechanism is naturally realized in several neutrino-portal scenarios with the secret neutrino interactions. We study possible evolution history of the hidden sector: the dark sector would undergo pure freeze-in production if the interactions between the dark-sector particles are negligible, while thermal equilibrium within the dark sector could occur if the reannihilation of the dark matter and the scalar mediator is rapid enough. We investigate the relic abundance in the freeze-in and dark freeze-out regimes, calculate evolution of the dark temperature, and study its phenomenological aspects on $\mathrm{BBN}$ and $\mathrm{CMB}$ constraints, the indirect-detection signature, as well as the potential to solve the small scale structure problem.

Keywords: Beyond Standard Model, Cosmology of Theories beyond the SM, Neutrino Physics

ARXIV EPRINT: 2005.01717

\footnotetext{
${ }^{1}$ Corresponding author.
} 


\section{Contents}

1 Introduction 1

2 The neutrino portal model $\quad 3$

3 The freeze-in production of dark matter 5

3.1 Pure freeze-in regime $\quad 7$

$\begin{array}{ll}3.2 & \text { Reannihilation regime } \\ & 11\end{array}$

$\begin{array}{lll}3.2 .1 & T_{D} \text { evolution } & 15\end{array}$

4 Model phenomenologies $\quad 16$

4.1 Dark scalar decay 16

$\begin{array}{lll}4.2 & \text { Indirect detection } & 18\end{array}$

$\begin{array}{lll}\text { 4.3 Possible solution to the small scale structure problem } & 19\end{array}$

5 Conclusion 23

A Cross sections and decay width $\quad 26$

$\begin{array}{ll}\text { B Energy transfer } & 27\end{array}$

\section{Introduction}

Nowadays, the existence of dark matter has been firmly supported by numerous astrophysical and cosmological observations. However, despite indisputable evidence for its existence, the nature of dark matter remains a mystery. Indeed, all the observational evidence, such as the observations on the galactic rotation curve [1], the Bullet Cluster [2] and the cosmic microwave background (CMB) [3], depends on the gravitational interaction of dark matter only. Due to the lack of knowledge on its non-gravitational interaction, the properties of dark matter, such as its mass, spin, its couplings to the Standard Model (SM), and whether it is composite or fundamental, remain unknown, nor do we know anything about its production mechanism. Understanding the nature of dark matter would not be so urgent if it plays an insignificant role in the universe. However, dark matter occupies roughly a quarter of the total energy density of the universe today, which is about five times the energy density of the content from the SM, it is the dominating species that governs the expansion of the universe in a large portion of the cosmic history, and it seeds the formation of structure which gives rise to the galaxies and the clusters of galaxies that we observe today. Therefore, resolving the mystery of dark matter is one of the biggest challenges facing particle physics, astrophysics and cosmology today.

Among the numerous attempts to tackle the problem of dark matter, the weakly interacting massive particle (WIMP) is perhaps the most studied dark-matter candidate. In the standard WIMP paradigm, the mass and the interaction strength between the WIMPs 
and the SM particles are often assumed to be at the weak scale. The WIMPs are assumed to be in thermal equilibrium with the radiation bath in the radiation dominated epoch, but freeze out of the thermal equilibrium when the interaction rate falls below the expansion rate. It is often referred to as the WIMP miracle that the combination of weak scale mass and coupling strength together with the thermal freeze-out mechanism successfully gives rise to the correct dark-matter relic abundance, $\Omega_{\mathrm{DM}} \simeq 0.26$. However, aside from its minimality and strong motivation from many models of the physics beyond the Standard Model (BSM), such as theories of supersymmetry, decades of null result in both direct and indirect detections, as well as collider searches, have made the standard WIMP scenarios more and more constrained [4]. Therefore, the current situation has invoked a lot of interest in other dark-matter scenarios.

Indeed, by modifying some of the defining features of the thermal WIMP paradigm, other viable dark-matter scenarios could also be constructed. For example, dark matter need not be produced via the thermal freeze-out mechanism. In fact, the interaction between dark matter and the SM particles could be very feeble such that the thermal equilibrium is never established between dark matter and the SM thermal bath. An equally motivated production mechanism suitable for this type of scenarios is the freeze-in mechanism [5-9] in which dark matter is produced gradually by the injection from the visible thermal bath. Typically, if dark matter is coupled to the thermal bath via renormalizable operators, its production is IR dominated by the temperature near its mass and thus insensitive to initial conditions. On the other hand, for non-renormalizable interactions [6], the production is sensitive to UV physics. Another approach is to modify the single-particle picture of most WIMP models. For instance, models from the non-minimal dark-sector framework often depend on the collective behavior of an entire ensemble of dark-sector particles [10-12]. One could also modify the standard $\Lambda$ cold dark matter $(\Lambda \mathrm{CDM})$ cosmology and consider dark-matter production in non-standard cosmologies, such as producing thermal dark matter during a matter dominated universe before the Big-Bang Nucleosynthesis (BBN), see [13, 14] for reviews and [15-17] for recent progress. By considering different possibilities, the relevant constraints and the potential signatures could be drastically different.

In this paper, we are focusing on the freeze-in production of dark matter. As mentioned above, the freeze-in scenario usually requires highly suppressed couplings between the SM and the dark sectors. Here, the question one can always ask is how such highly suppressed couplings are naturally generated. Previously, the Higgs portal has been studied to explain the current dark matter relic density through freeze-in, where the couplings are suppressed by the small vacuum expectation value (VEV) of the new Higgs field (see [7] for review and references in it). However, the smallness of the new Higgs VEV remains mysterious in general. On the other hand, a counterexample is the dark photon model [18, 19], wherein successful freeze-in is realized by a small kinetic mixing between the SM photon and the dark photon. Such a tiny kinetic mixing can be naturally explained by loop diagrams of heavy fields charged under both the $\mathrm{U}(1)_{\mathrm{Y}}$ and the $\mathrm{U}(1)_{\text {dark }}$ groups.

In this paper, we consider another natural realization of the freeze-in scenario in which the dark-matter particles are gradually populated through a neutrino portal. To be spe- 
cific, we consider a hidden sector consisting of a dark scalar mediator $\phi$ and a fermionic dark matter $\chi$, and the hidden sector is only connected to the SM via a neutrino portal. Typically, there are two kinds of neutrino-portal dark-matter models - the $t$-channel models with the $\nu-\chi-\phi$ interaction [20-33], and the $s$-channel models with the $\nu-\nu-\phi$ interaction $[34,35]$, in the freeze-out scenarios. While the $t$-channel models have been investigated in details in recent years, in this paper, we consider the $s$-channel models in which the dark matter $\chi$ only interacts with the SM neutrinos through an $s$-channel exchange of $\phi$ in the freeze-in regime. We find that, the small coupling between the hidden sector and the SM neutrinos can be naturally generated via two types of UV models, the Type-I seesaw model and the Minimal Majoron model.

Though the freeze-in scenario lacks the direct detection signal due to its feeble couplings to the SM sector, we argue that the freeze-in scenario could give rise to much richer cosmological history compared to the freeze-out scenario. In particular, while the darkmatter production depends only on the energy injection from the SM thermal bath if the interaction rate between $\chi$ and $\phi$ is negligible, the reannihilation of $\chi$ and $\phi$ could also establish a period of thermal equilibrium within the dark sector if such interaction is sufficiently rapid [7-9, 36-39]. In the latter case, the dark-sector particles would eventually break away from the thermal equilibrium either by directly freezing out of the dark thermal equilibrium as their interaction rate drops below the expansion rate, or by entering a phase called the Quasi-Static equilibrium (QSE) [36] in which the annihilation rate of $\chi$ is much larger than that of $\phi$ and is balanced with the injection from the SM sector before finally freezing out of it. Ultimately, the relic abundance of the dark matter is sensitive to the differences in the evolution history.

A sizable interaction strength within the dark sector would allow rapid annihilations of $\chi$ inside dark-matter halos at the present epoch. Such late-time annihilations of $\chi$ followed by a rapid decay of $\phi$ could produce a substantial amount of neutrinos and thus would be constrained by the current neutrino observations. In addition, a sufficiently large coupling between $\chi$ and $\phi$ could be exploited to solve problems on small scale structure at late time, making our model a potentially viable candidate for the self-interacting dark matter (SIDM, see e.g. ref. [40] for review and the references in it).

This paper is organized as follows. In section 2, we set up the neutrino-portal darkmatter model and discuss several examples for UV completion. In section 3, we study the evolution of the dark sector in different regimes and discuss the consequence of having different evolution history. In section 4 , we discuss the phenomenological aspects of our model, including the constraints on the decay of the dark scalar $\phi$, the potential for our model to solve the small scale structure problems, and the indirect-detection constraints. Finally, we conclude in section 5.

\section{The neutrino portal model}

In the $s$-channel neutrino model, we consider a dark sector which consists of a Dirac fermionic $\chi$ and a light scalar mediator $\phi$. While the dark-matter candidate $\chi$ is directly coupled to $\phi$ only, the mediator $\phi$ is in addition coupled to the SM neutrinos. To be specific, 
the effective Lagrangian for a dark sector described above can be in general written as

$$
\begin{aligned}
\mathcal{L} & =\mathcal{L}_{\text {kin }}+\mathcal{L}_{\text {int }}, \\
\mathcal{L}_{\text {kin }} & =\frac{1}{2}\left(\partial_{\mu} \phi \partial^{\mu} \phi-m_{\phi}^{2} \phi^{2}\right)+\bar{\chi}\left(i \not \partial-m_{\chi}\right) \chi, \\
\mathcal{L}_{\text {int }} & =-\phi \bar{\chi}\left(g_{\chi}+i \bar{g}_{\chi} \gamma^{5}\right) \chi-\phi \bar{\nu}_{i}\left(g_{\nu}^{i j}+i \bar{g}_{\nu}^{i j} \gamma^{5}\right) \nu_{j},
\end{aligned}
$$

where $g_{\chi}$ and $\bar{g}_{\chi}$ are the couplings between the mediator $\phi$ and the dark matter $\chi$, while $g_{\nu}^{i j}$ and $\bar{g}_{\nu}^{i j}$ are the effective couplings of $\phi$ to the SM neutrinos in the flavor basis with the superscript $i$ and $j$ being the flavor index. In our model, we do not assume any nonvanishing VEV for the $\phi$ field. Consequently, 1) $\phi$ does not mix with the SM Higgs boson and could thus evade all the collider constraints; 2) the only way for the dark sector to interact with the SM sector is through the light mediator $\phi$. However, it is possible that the Higgs VEV might contribute to the mass of $\phi$, which we implicitly include in the $m_{\phi}$ term.

To produce dark matter through freeze-in in the early universe, we generically require the couplings $g_{\nu}^{i j}$ and $\bar{g}_{\nu}^{i j}$ to be very tiny in order to avoid establishing thermal equilibrium between the dark and the SM sectors. Such small couplings could be naturally generated through appropriate UV models. In what follows, we shall discuss the UV completion for the general Lagrangian above in two limiting scenarios - the one with a pure scalar $\phi$ and only pure-scalar interactions; and the one in which $\phi$ is a pseudoscalar, and only pseudoscalar interactions exist. We shall refer to these two scenarios as Scenario $I$ and Scenario II. The interacting Lagrangian in eq. (2.3) can be rewritten by setting $\bar{g}_{\chi(\nu)}=0$ or $g_{\chi(\nu)}=0$ for the two scenarios, respectively:

$$
\mathcal{L}_{\text {int }}= \begin{cases}-g_{\chi} \phi \bar{\chi} \chi-g_{\nu}^{i j} \phi \bar{\nu}_{i} \nu_{j} & (\text { Scenario I }), \\ -i \bar{g}_{\chi} \phi \bar{\chi} \gamma^{5} \chi-i \bar{g}_{\nu}^{i j} \phi \bar{\nu}_{i} \gamma^{5} \nu_{j} & \text { (Scenario II) }\end{cases}
$$

For Scenario I, we are motivated by the type-I seesaw and introduce the right-handed neutrinos $N$ in addition to the scalar $\phi$. We assume the masses $M_{N} \gg M_{\mathrm{EW}}$ with $M_{\mathrm{EW}}$ being the electroweak scale. At high energy scale, the Lagrangian can be written as

$$
\mathcal{L}_{\text {int }} \supset g_{H} \bar{L} \tilde{H} N+g_{\phi} \overline{N^{c}} N \phi+\text { h.c. },
$$

where $\tilde{H} \equiv i \sigma_{2} H^{*}$. Around the electroweak scale, one can integrate out the heavy fields $N_{i}$ and obtain the dimension- 6 operators: ${ }^{1}$

$$
\mathcal{L}_{\text {int }} \supset \frac{1}{M_{N}^{2}} \phi(\bar{L} \tilde{H})\left(\tilde{H}^{T} L^{c}\right) .
$$

After electroweak spontaneous symmetry breaking, tree level interactions between the scalar $\phi$ and the SM neutrinos can be generated as follows:

$$
\frac{1}{M_{N}^{2}} \phi(\bar{L} \tilde{H})\left(\tilde{H}^{\dagger} L\right) \rightarrow g_{\nu} \phi \bar{\nu} \nu, \quad \text { with } g_{\nu} \sim \frac{v^{2}}{M_{N}^{2}},
$$

\footnotetext{
${ }^{1}$ We shall suppress the neutrino flavor index from now on, if not otherwise mentioned.
} 
where $v$ is the SM Higgs VEV. Note that since we assume $M_{N} \gg M_{\mathrm{EW}}, g_{\nu}$ is naturally suppressed.

For Scenario II, the $\phi-\nu-\nu$ interaction for a pseudoscalar $\phi$ could be generated in the so-called minimal Majoron model [41-44]. In this type of model, we still introduce three right-handed neutrinos $N_{i}$ but in addition a SM-singlet complex scalar field $\Phi$ above the electroweak scale. At high energy scale, the interacting Lagrangian can be written as

$$
\mathcal{L}_{\text {int }} \supset g_{\Phi} \overline{N^{c}} N \Phi-g_{H} \bar{L} \tilde{H} N+\text { h.c. } .
$$

We then assume that $\Phi$ develops a VEV and spontaneously breaks a global U(1) symmetry at a scale higher than $M_{\mathrm{EW}}$. Therefore, we can write $\Phi=\varphi+v_{\Phi}+i \phi$, where $v_{\Phi}$ is the VEV, the pseudoscalar $\phi$ is the Nambu-Goldstone boson, and we assume $v_{\Phi} \gg v$. We further assume that the $\mathrm{U}(1)$ symmetry is not exact, but explicitly broken by a soft-breaking mass term in the potential of $\Phi$. As a result, the pseudoscalar $\phi$ acquires a small mass and becomes a pseudo Nambu-Goldstone boson. After the symmetry breaking, first term in eq. (2.8) generates the Majorana mass term and the interacting Lagrangian becomes

$$
\mathcal{L}_{\text {int }} \supset-\frac{M_{N}}{2} \bar{N} N-i g_{\Phi} \phi \bar{N} \gamma_{5} N-g_{H} \bar{L} \tilde{H} N
$$

with $M_{N}=g_{\Phi} v_{\Phi}$, where we have transform $N$ to the four component notation for a Majorana field such that $N^{c}=N$. We can then integrate out the heavy filed $N$, and the effective Lagrangian can be expressed as

$$
\mathcal{L}_{\text {int }} \supset i \frac{g_{\Phi} g_{H}^{2}}{M_{N}^{2}} \phi(\bar{L} \tilde{H}) \gamma^{5}\left(\tilde{H}^{T} L^{c}\right)+\text { h.c. } .
$$

Finally, after the electroweak spontaneous symmetry breaking,

$$
\mathcal{L}_{\text {int }} \supset-i \bar{g}_{\nu} \phi \bar{\nu} \gamma^{5} \nu, \quad \text { with } \bar{g}_{\nu} \sim \frac{v^{2}}{M_{N}^{2}} \sim \frac{v^{2}}{g_{\Phi}^{2} v_{\Phi}^{2}} .
$$

Again, $\bar{g}_{\nu}$ is naturally suppressed. We point out that it has also been studied when the pNGB $\phi$ becomes a dark-matter candidate in refs. [45-50].

Having demonstrated how Scenario I and Scenario II could be generated from UV theories, in what follows, we shall simply use the effective Lagrangian in eq. (2.4) when discussing production of dark matter. Note that, in the above discussion, we choose not to specify the coupling strength between $\chi$ and $\phi$. As a consequence, while a highly suppressed $g_{\nu}$ or $\bar{g}_{\nu}$ could ensure a sufficiently feeble interaction between the SM sector and the dark sector during the dark-matter production, by tuning the couplings $g_{\chi}$ or $\bar{g}_{\chi}$, we are able to study interesting dark-sector dynamics and phenomenologies in several different regimes. To conclude this section, we list all the Feynman diagrams relevant for our scenarios in figure 1.

\section{The freeze-in production of dark matter}

As we have discussed in the previous sections, we are interested in the regime where the interaction between the dark-matter particles and the SM neutrinos is too feeble to ever 


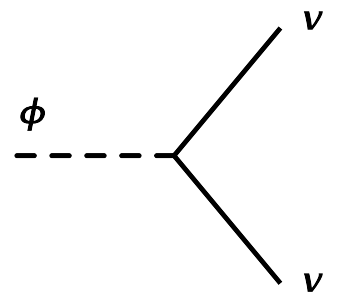

(a)

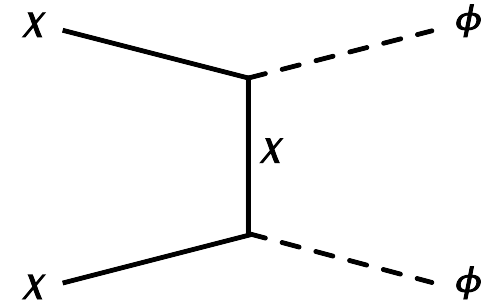

(b)

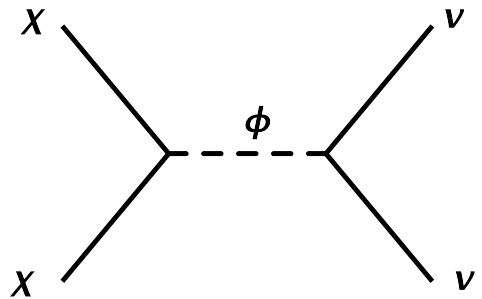

(c)

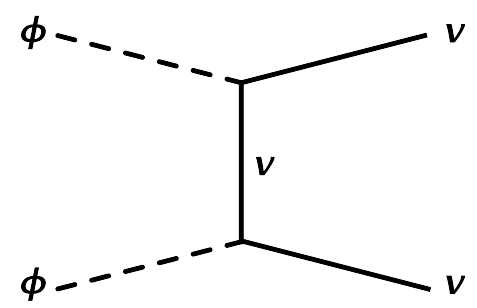

(d)

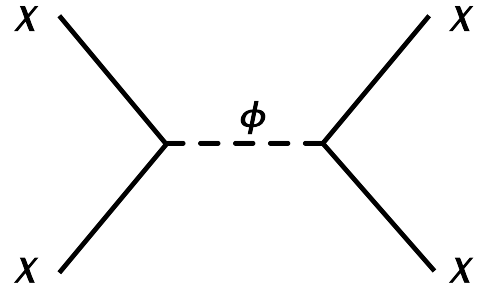

(e)

Figure 1. Relevant Feynman diagrams in our study.

establish thermal equilibrium between them, and the dark sector is mainly populated by a gradual injection of energy from the SM thermal bath, i.e. via the freeze-in mechanism. Since the couplings in eq. (2.3) are all renormalizable, our scenario falls in the category of IR freeze-in. Similar to the freeze-out scenario, it is well know that the final yield of darkmatter particles is insensitive to the initial condition in IR freeze-in scenarios. Therefore, without loss of generality, we shall also assume that the initial energy density of the darksector particles is negligible. To be concrete, the evolution of dark-matter number density is determined by the following Boltzmann equation

$$
\frac{d n_{\chi}}{d t}=-3 H n_{\chi}-\left(n_{\chi}^{2}-n_{\chi}^{\mathrm{eq}}(T)^{2}\right)\left\langle\sigma_{\chi \chi \rightarrow \nu \nu} v\right\rangle_{T}-n_{\chi}^{2}\left\langle\sigma_{\chi \chi \rightarrow \phi \phi} v\right\rangle_{T_{\chi}}+n_{\phi}^{2}\left\langle\sigma_{\phi \phi \rightarrow \chi \chi} v\right\rangle_{T_{\phi}},
$$

where $T$ refers to the temperature of the SM thermal bath, and $T_{\chi, \phi}$ are the temperatures that the dark-sector particles $\chi$ and $\phi$ might have during their evolution which are not necessarily identical. In the equation above, we have implicitly assumed that the phase-space distribution of $\chi$ and $\phi$ takes the form $f_{\chi, \phi} \sim e^{-\left(E-\mu_{\chi, \phi}\right) / T_{\chi, \phi}}$ with their own temperature and chemical potential. Besides, the condition of detailed balance, $f_{\nu}^{\mathrm{eq}}\left(p_{\nu_{1}}\right) f_{\nu}^{\mathrm{eq}}\left(p_{\nu_{2}}\right)=$ $f_{\chi}^{\mathrm{eq}}\left(p_{\chi_{1}}\right) f_{\chi}^{\mathrm{eq}}\left(p_{\chi_{2}}\right)$, is applied to convert $n_{\nu}^{\mathrm{eq}}(T)^{2}\left\langle\sigma_{\nu \nu \rightarrow \chi \chi}\right\rangle_{T}$ - the term that describes the production of dark matter from neutrino annihilation, into $n_{\chi}^{\mathrm{eq}}(T)^{2}\left\langle\sigma_{\chi \chi \rightarrow \nu \nu}\right\rangle_{T}$ which involves only the number density of $\chi$ at the temperature of the SM thermal bath. In general, if the scattering rates are too slow, $\phi$ or $\chi$ might not be able to reach kinetic equilibrium - they might not have a thermal distribution with a well defined temperature. Nevertheless, we shall keep using the thermally averaged cross-section assuming that it is a good approximation when the typical kinetic energy of the dark-sector particles is $T_{\chi, \phi}$. 


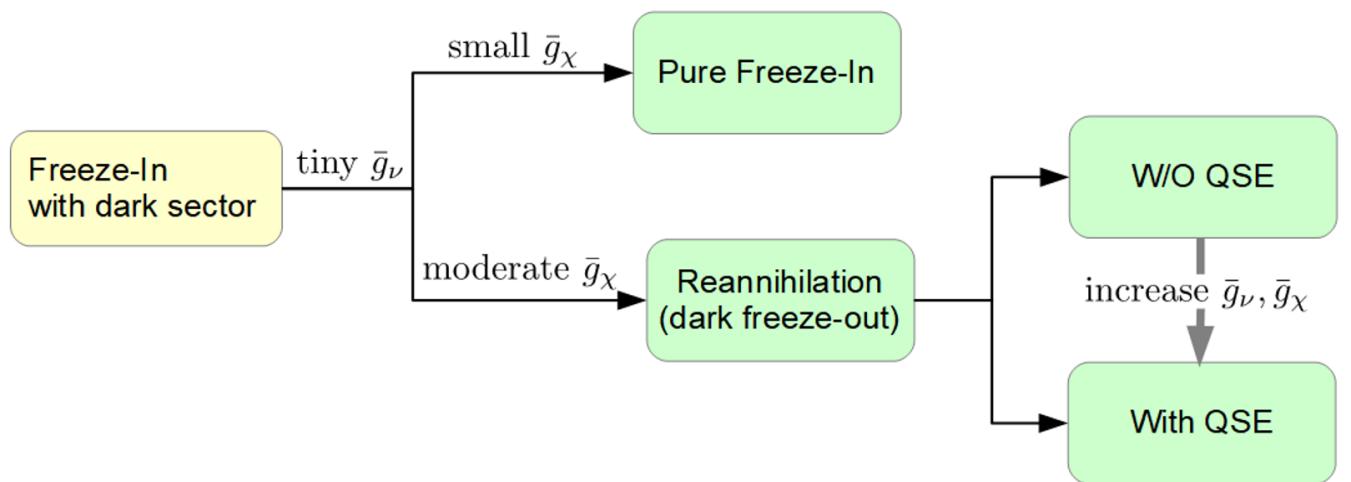

Figure 2. The classification of production mechanism for our freeze-in dark matter model. $\bar{g}_{\chi}$ and $\bar{g}_{\nu}$ are the pseudoscalar couplings of the mediator $\phi$ to the dark matter particle $\chi$ and the SM neutrino respectively. The quasi-static equilibrium (QSE) [36] refers to the situation in which the $\chi \chi \rightarrow \phi \phi$ process greatly exceeds its inverse process due to the injection of $\chi$ from the thermal bath and the injection of $\chi$ is balanced by its annihilation into $\phi$.

As it is already discussed in refs. [8, 9, 36-38], depending on $g_{\chi}$ and/or $\bar{g}_{\chi}$ and the dark-sector number densities, the production of dark-sector particles can be categorized into two different regimes:

- The pure freeze-in regime, where the couplings between $\chi$ and $\phi$ are so small that interactions between them are negligible. As a result, $\chi$ and $\phi$ can never reach thermal equilibrium.

- The reannihilation regime, where the couplings $g_{\chi}$ and/or $\bar{g}_{\chi}$ are large enough that the annihilations process $\chi \chi \leftrightarrow \phi \phi$ starts to play a significant role in the evolution of $n_{\chi}$ and $n_{\phi}$. This could also allow $\chi$ and $\phi$ to reach thermal equilibrium during a certain period of time in the evolution of the universe with a common temperature denoted as $T_{D}$ which is smaller than the temperature of the SM thermal bath $T$.

In what follows, we describe these two regimes in detail while focusing on Scenario II in which $\phi$ is a pseudoscalar case, as the discussion can be easily carried over to Scenario I in which $\phi$ is a pure scalar. The only difference between the two scenarios is that while the annihilation cross-section for $\chi \chi \rightarrow \nu \nu$ is $s$-wave in the Scenario II, it is $p$-wave suppressed in the Scenario I which leads to a lower efficiency in dark-matter production. We shall see this in more details later. We also associate the figure 2 for a summary for the different regime with the corresponding scale of the relevant couplings.

\subsection{Pure freeze-in regime}

As mentioned above, in the pure freeze-in regime, not only the dark matter is decoupled from the SM thermal bath, the thermal equilibrium between dark-sector constituents $\chi$ and $\phi$ is also never reached. Therefore, during the production of dark-matter particles $\chi$, one can make the following approximations: 1 ) the number density $n_{\chi}$ is so small that the annihilation process $\chi \chi \rightarrow \nu \nu$ can be safely neglected; 2) the particle exchange between 
dark-sector species $\phi \phi \leftrightarrow \chi \chi$ is also negligible. With these approximations, the Boltzmann equation eq. (3.1) can be approximated as

$$
\frac{d n_{\chi}}{d t} \approx-3 H n_{\chi}+n_{\chi}^{\mathrm{eq}}(T)^{2}\left\langle\sigma_{\chi \chi \rightarrow \nu \nu} v\right\rangle_{T},
$$

where $T$ represents the temperature of the SM thermal bath, and we have used the condition $n_{\chi}^{\mathrm{eq}}(T) \gg n_{\chi}(T)$.

To connect with the observed dark-matter relic density today, it is often convenient to use entropy conservation $\tilde{s} a^{3}=$ const. where $\tilde{s}$ is the entropy density and $a$ is the scale factor, and define the comoving number density or the yield of dark matter $Y_{\chi} \equiv n_{\chi} / \tilde{s}$ which eventually becomes a constant after the freeze-in process concludes. Therefore, to reproduce the expected present-day dark-matter relic density, the final yield $Y_{\chi}\left(T_{f}\right)$ is required to be

$$
Y_{\chi}\left(T_{f}\right)=Y_{\chi}\left(T_{\text {now }}\right) \approx \frac{\rho_{\text {crit }} \Omega_{\chi}}{m_{\chi} \tilde{s}\left(t_{\text {now }}\right)} \approx 4.14 \times 10^{-10} \times\left(\frac{\mathrm{GeV}}{m_{\chi}}\right) .
$$

The above expression can thus be used to constrain our model parameters. In what follows, we shall estimate the yield from the pure freeze-in process. We first note that

$$
\sigma_{\chi \chi \rightarrow \nu \nu} v_{\mathrm{M} \varnothing \mathrm{l}}=\frac{3 \bar{g}_{\chi}^{2} \bar{g}_{\nu}^{2} s^{2}}{64 \pi\left[\left(s-m_{\phi}^{2}\right)^{2}+m_{\phi}^{2} \Gamma_{\phi}^{2}\right] E_{\chi_{1}} E_{\chi_{2}}},
$$

where $\Gamma_{\phi}$ is the decay width of $\phi, s$ is the Mandelstam variable, and $E_{\chi_{1}}, E_{\chi_{2}}$ represent the energy of the dark-matter particles in the annihilation process. In the high-temperature regime $\left(T \gg m_{\chi}\right)$, most of the annihilations take place with $s \gg 4 m_{\chi}^{2}$, whereas in the low-temperature regime $\left(T \ll m_{\chi}\right)$, typically $s \sim 4 m_{\chi}^{2}$. Taking these approximations, the thermally averaged cross-section can be expressed as follows assuming $m_{\chi} \gg m_{\phi}$ :

$$
\left\langle\sigma_{\chi \chi \rightarrow \nu \nu} v_{\mathrm{M} \varnothing \mathrm{l}}\right\rangle_{T} \approx \begin{cases}\frac{3 \bar{g}_{\chi}^{2} \bar{g}_{\nu}^{2}}{128 \pi\left\langle E_{\chi}\right\rangle^{2}} \simeq 7.5 \times 10^{-4} \times \frac{\bar{g}_{\chi}^{2} \bar{g}_{\nu}^{2}}{T^{2}}, & T \gg m_{\chi} \\ \frac{3 \bar{g}_{\chi}^{2} \bar{g}_{\nu}^{2}}{64 \pi\left\langle E_{\chi}\right\rangle^{2}} \simeq 0.015 \times \frac{\bar{g}_{\chi}^{2} \bar{g}_{\nu}^{2}}{m_{\chi}^{2}}, & T \ll m_{\chi},\end{cases}
$$

where we have applied $\left\langle E_{\chi}\right\rangle=\rho_{\chi} / n_{\chi} \simeq 3.15 T$ when $T \gg m_{\chi}$ and $\left\langle E_{\chi}\right\rangle \simeq m_{\chi}$ when $T \ll m_{\chi}$. Assuming that $f_{\chi}=\exp \left(-E_{\chi} / T\right)$, we further obtain the expressions for the annihilation rate:

$$
n_{\chi}^{\mathrm{eq}}\left\langle\sigma_{\chi \chi \rightarrow \nu \nu} v\right\rangle_{T} \simeq\left\{\begin{array}{ll}
1.4 \times 10^{-4} \bar{g}_{\chi}^{2} \bar{g}_{\nu}^{2} T, & T \gg m_{\chi} \\
1.9 \times 10^{-3} \bar{g}_{\chi}^{2} \bar{g}_{\nu}^{2} T \times \sqrt{\frac{T}{m_{\chi}}} \exp \left(-\frac{m_{\chi}}{T}\right), & T \ll m_{\chi}
\end{array} .\right.
$$

In the pure freeze-in regime, since the $\nu \nu \rightarrow \chi \chi$ process is the only production channel for the dark-matter particles, the condition that the dark matter never reaches thermal equilibrium with the SM generally requires that

$$
n_{\chi}^{\mathrm{eq}}\left\langle\sigma_{\chi \chi \rightarrow \nu \nu} v\right\rangle_{T}<H
$$


where $H$ is the Hubble expansion rate. In the radiation dominated epoch, we have

$$
H \simeq \sqrt{\frac{\rho_{R}}{3 M_{P}^{2}}}=\sqrt{\frac{\pi^{2}}{90}} g_{\star}^{1 / 2}(T) \frac{T^{2}}{M_{P}},
$$

where $g_{\star}$ is the effective numbers of relativistic degrees of freedom for the energy density, and $M_{P}$ is the reduced Planck mass. Since the number density in eq. (3.5) is always exponentially suppressed in the non-relativistic regime, we simply require eq. (3.6) to be satisfied in the relativistic regime, which implies

$$
\bar{g}_{\chi} \bar{g}_{\nu} \lesssim \mathcal{O}\left(10^{-8}\right) \times\left(\frac{m_{\chi}}{\mathrm{GeV}}\right)^{1 / 2},
$$

if $g_{\star}^{1 / 2}(T) \sim \mathcal{O}(10)$.

To proceed further with the estimate, we rewrite the eq. (3.2) as

$$
\frac{d Y_{\chi}}{d T} \approx-\frac{s\langle\sigma v\rangle}{H T} Y_{\chi}^{\mathrm{eq}}(T)^{2},
$$

which we can directly integrate from a large initial temperature $T_{i} \gg m_{\chi}$ to the present-day temperature $T_{\text {now }}$ :

$$
\int_{Y_{\chi}^{i}}^{Y_{\chi}^{\text {now }}} d Y_{\chi} \approx-\int_{T_{\text {now }}}^{T_{i}} d T \frac{s\langle\sigma v\rangle}{H T} Y_{\chi}^{\mathrm{eq}}(T)^{2}
$$

Using our previous discussion, the integrand

$$
\frac{s\langle\sigma v\rangle}{H T} Y_{\chi}^{\mathrm{eq}}(T)^{2} \propto\left\{\begin{array}{ll}
1 / T^{2}, & T \gg m_{\chi} \\
e^{-2 m_{\chi} / T} / T^{3}, & T \ll m_{\chi}
\end{array} .\right.
$$

Obviously, the Boltzmann suppression factor which appears in the non-relativistic regime suggests that the contribution to the entire integral from this part, i.e. $m_{\chi}>T \geq T_{\text {now }}$, is always subleading. At the same time, the $1 / T^{2}$ behavior in the relativistic regime also indicates that the production of dark matter is very small when $T \gg m_{\chi}$. In fact, most of the contribution to the integral in eq. (3.10) actually comes from the part where $T \sim m_{\chi}$. Therefore, we find as expected that the dark-matter production in the pure freeze-in regime is largely insensitive to the initial conditions.

Based on our discussions, we can then make following approximations and roughly estimate the final yield:

$$
Y_{\chi}^{\text {now }} \approx \int_{T_{i}}^{m_{\chi}} d T \frac{s\langle\sigma v\rangle}{H T} Y_{\chi}^{\mathrm{eq}}(T)^{2} \approx 2.1 \times 10^{14} \times \frac{\bar{g}_{\chi}^{2} \bar{g}_{\nu}^{2}}{g_{\star}^{1 / 2}\left(m_{\chi}\right) g_{\star, s}\left(m_{\chi}\right)}\left(\frac{\mathrm{GeV}}{m_{\chi}}\right),
$$

in which $g_{\star, s}$ is the effective numbers of relativistic degrees of freedom for entropy density. For $m_{\chi} \sim \mathcal{O}(\mathrm{GeV})$, the denominator $g_{\star}^{1 / 2} g_{\star, s} \sim 10^{3}$. Comparing with eq. (3.3), one generally need $\bar{g}_{\chi} \bar{g}_{\nu} \sim \mathcal{O}\left(10^{-11}\right)$ to obtain the correct dark-matter relic abundance.

Examples of the pure freeze-in scenario for both the pseudoscalar (upper panels) and scalar (lower panels) cases are shown in figure 3, in which we have chosen to plot against 

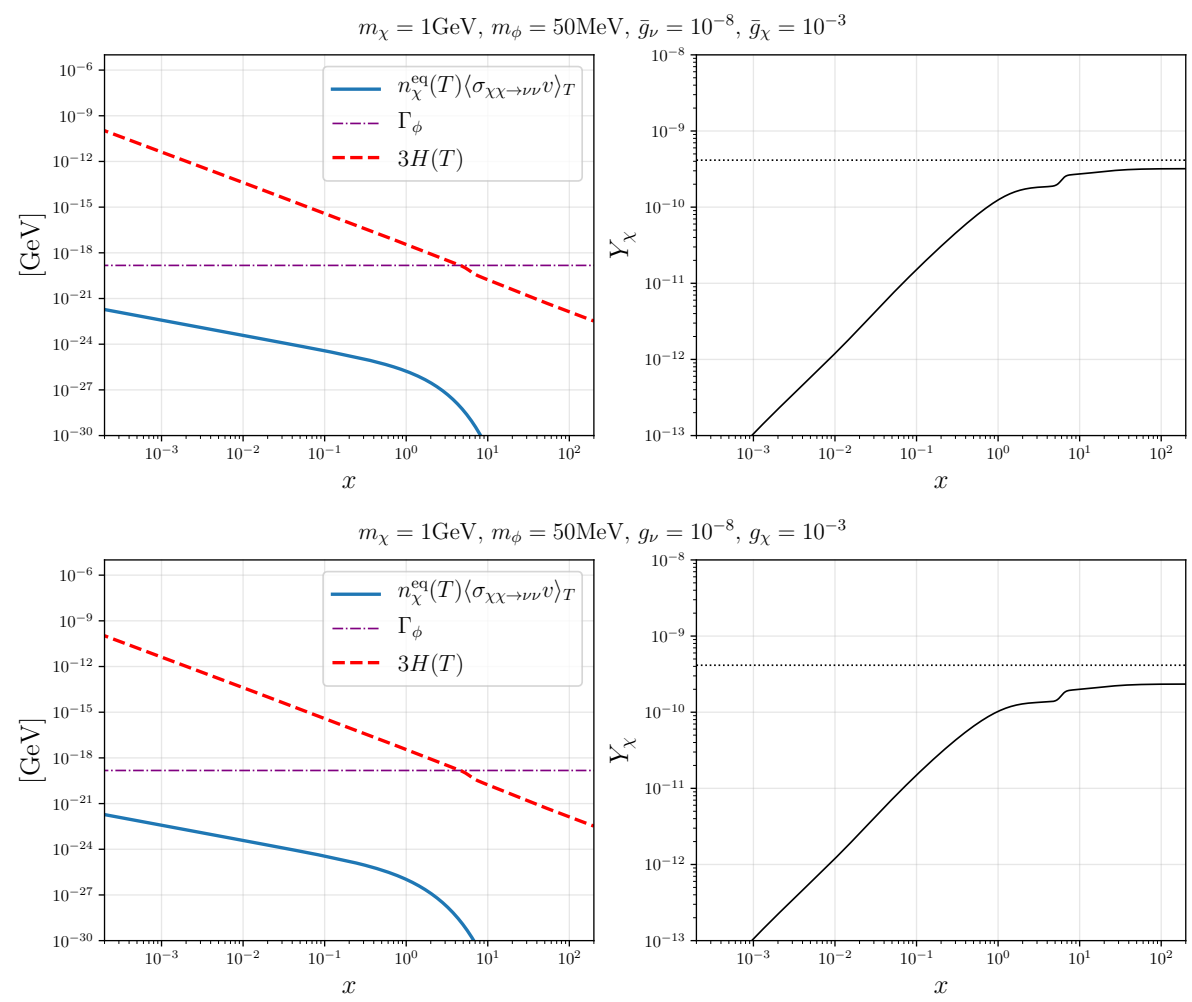

Figure 3. Examples in the pure freeze-in regime. The upper panels correspond to Scenario $I I$ where $\phi$ is a pseudoscalar, and the lower panels correspond to Scenario $I$ where $\phi$ is a pure scalar. In the left panels, the blue curve shows the evolution of the dark-matter production rate $n_{\chi}^{\mathrm{eq}}(T)\left\langle\sigma_{\chi \chi \rightarrow \nu \nu} v\right\rangle_{T}$, whereas the dash-dotted purple line is the decay rate of $\phi$. The dashed red curve is the evolution of the Hubble expansion rate $H$. In the right panels, we show the evolution of $Y_{\chi}$ as a function of $x \equiv m_{\chi} / T$. The dotted horizontal line indicates the observed relic density of the dark matter today.

$x \equiv m_{\chi} / T$. In the left panels, we show a comparison between the expansion rate $H$ and the dark-matter production rate $n_{\chi}^{\mathrm{eq}}(T)\left\langle\sigma_{\chi \chi \rightarrow \nu \nu} v\right\rangle_{T}$. As expected, the dark-matter production rate is always smaller than $H$. However, despite the fact that it is always decreasing as the SM temperature drops, it is closest to $H$ at $T \sim m_{\chi}$, which supports the approximation we use in eq. (3.12). After the temperature drops below $m_{\chi}$, a quick down turn appears as the neutrinos no longer have enough energy to create the dark-matter particles. As a result, we see in the right panel that the yield grows monotonically from a vanishingly small initial value, and then asymptotes to its maximum near $T \sim m_{\chi}$. Note that the sudden jump for both $H$ and $Y_{\chi}$ around $x \sim 5-10$ is not a numerical artifact but merely due to the sudden change in $g_{\star}$ and $g_{\star, s}$ when the temperature drops to $T \sim \mathcal{O}(100) \mathrm{MeV}$, i.e., when the QCD phase transition occurs.

Comparing the pseudoscalar with the pure scalar cases, it is obvious that for couplings of the same size, the final yield in the latter is slightly smaller. This is simply because the factor $s^{2}$ in the numerator of the thermally averaged cross-section in eq. (3.4) becomes $s(s-$ $\left.4 m_{\chi}^{2}\right)$ in Scenario I (see eq. (A.1) for more details), which results in a $p$-wave suppression 
in the production rate as $T$ approaches $m_{\chi}$. Interestingly, in thermal freeze-out scenarios, the $p$-wave suppression typically leads to an earlier freeze-out which, by contrast, increases rather than decreases the final yield of dark matter.

\subsection{Reannihilation regime}

In the pure freeze-in regime, the interactions between the dark-sector particles are so weak such that both $\chi$ and $\phi$ barely annihilate. As a consequence, the $\chi \chi \leftrightarrow \phi \phi$ process has little impact on the evolution of dark-matter number density. However, it is also possible that while the entire dark sector is decoupled from the SM sector, the annihilations of $\chi$ and $\phi$ proceed with a non-negligible rate. Indeed, since the interaction strength between $\chi$ and $\phi$ is governed by the coupling $\bar{g}_{\chi}$ in Scenario II (or $g_{\chi}$ in Scenario I), by increasing $\bar{g}_{\chi}$ while adjusting $\bar{g}_{\nu}$ accordingly in order to prevent thermalization with the SM sector, as required in eq. (3.8), the dark sector could establish its own thermal equilibrium for a period of time in the history of the universe as it is populated by the SM thermal bath. This thermal equilibrium is characterized by a common temperature $T_{D}$ for both $\chi$ and $\phi$. In this situation, eq. (3.1) can be rewritten as

$$
\frac{d n_{\chi}}{d t} \approx-3 H n_{\chi}+n_{\chi}^{\mathrm{eq}}(T)^{2}\left\langle\sigma_{\chi \chi \rightarrow \nu \nu} v\right\rangle_{T}-\left(n_{\chi}^{2}-n_{\chi}^{\mathrm{eq}}\left(T_{D}\right)^{2}\right)\left\langle\sigma_{\chi \chi \rightarrow \phi \phi} v\right\rangle_{T_{D}},
$$

in which the thermally averaged cross-section for $\chi \chi \leftrightarrow \phi \phi$ is evaluated at $T_{D}$.

The thermal equilibrium in the dark sector requires the interaction rate between the dark-sector particles $n_{\chi}^{\mathrm{eq}}\left(T_{D}\right)\left\langle\sigma_{\chi \chi \rightarrow \phi \phi}\right\rangle_{T_{D}}>H(T)$. For a sizable cross-section $\sigma_{\chi \chi \rightarrow \phi \phi}$, this condition could be immediately realized if the initial particle number density $n_{\chi}$ or $n_{\phi}$ is large enough. By contrast, if the initial number density is small, the dark sector could start without thermal equilibrium but slowly build it up as the SM sector keeps populating the dark-sector particles. Once the thermal equilibrium is established within the dark sector, one would expect the number density $n_{\chi}$ to track $n_{\chi}^{\mathrm{eq}}\left(T_{D}\right)$, since the last term in the Boltzmann equation above always tends to bring back any deviation from the thermal equilibrium. This is indeed true if the second term on the right-hand side of eq. (3.13) is not large enough such that the $\chi \chi \rightarrow \phi \phi$ process can always be balanced by the $\phi \phi \rightarrow \chi \chi$ process. However, if this SM source term is sufficiently large, $n_{\chi}$ might be able to grow significant larger than $n_{\chi}^{\mathrm{eq}}\left(T_{D}\right)$. Therefore, the annihilation rate of $\chi$ could overwhelm that of $\phi$, and this could occur even when the latter is still larger than the Hubble rate. In this situation, eq. (3.13) can be approximated as

$$
\frac{d n_{\chi}}{d t} \approx-3 H n_{\chi}+n_{\chi}^{\mathrm{eq}}(T)^{2}\left\langle\sigma_{\chi \chi \rightarrow \nu \nu} v\right\rangle_{T}-n_{\chi}^{2}\left\langle\sigma_{\chi \chi \rightarrow \phi \phi} v\right\rangle_{T_{D}} .
$$

The equation above simply means that while the SM thermal bath is always sourcing the dark matter $\chi$, the annihilation of $\chi$ always tends to deplete this particle injection. As a result, the QSE phase often occurs in which the last two terms in eq. (3.13) are balanced against each other [36]. To be precise, the QSE phase is defined by

$$
Y_{\chi}(T) \approx Y_{\chi}^{\mathrm{QSE}}(T) \equiv Y_{\chi}^{\mathrm{eq}}(T) \sqrt{\frac{\left\langle\sigma_{\chi \chi \rightarrow \nu \nu} v\right\rangle_{T}}{\left\langle\sigma_{\chi \chi \rightarrow \phi \phi} v\right\rangle_{T_{D}}}} .
$$


The dark thermal equilibrium or the QSE persists until $n_{\chi}(T)\left\langle\sigma_{\chi \chi \rightarrow \phi \phi}\right\rangle_{T_{D}} \lesssim H(T)$, and then the dark matter experiences a dark freeze-out. However, unlike the standard thermal freeze-out scenario, the comoving number density of the dark matter might not be fixed at its value around the dark freeze-out, because the production of $\chi$ from the SM thermal bath could still make a significant contribution to the final yield even after the dark freeze-out. In any case, differences caused by having different initial number densities for $\chi$ or $\phi$ would eventually be washed out by the thermalization within the dark sector - the freeze-in mechanism is also independent of initial conditions in the reannihilation regime.

As mentioned above, eq. (3.13) depends on the dark-sector temperature $T_{D}$, which, unlike the SM temperature, is not known a priori. In order to find $T_{D}$, we numerically solve the Boltzmann equation for the total energy density in the dark sector:

$$
\begin{aligned}
\frac{d \rho_{D}}{d t}= & -3 H\left(P_{D}+\rho_{D}\right)+2 n_{\nu}^{\mathrm{eq}}(T)^{2} \mathcal{P}_{\nu \nu \rightarrow \chi \chi}(T)+2 n_{\nu}^{\mathrm{eq}}(T)^{2} \mathcal{P}_{\nu \nu \rightarrow \phi \phi}(T) \\
& +n_{\nu}^{\mathrm{eq}}(T)^{2} \mathcal{P}_{\nu \nu \rightarrow \phi}(T)-n_{\phi} \mathcal{P}_{\phi \rightarrow \nu \nu}\left(T_{D}\right),
\end{aligned}
$$

where the energy density $\rho_{D} \equiv \rho_{\chi}+\rho_{\phi}$, the pressure $P_{D} \equiv P_{\chi}+P_{\phi}$, and the thermally averaged energy transfer rate $\mathcal{P}_{a \cdots \rightarrow i \ldots}$ gives the energy transferred to or from the dark sector through the process $a+\cdots \rightarrow i+\ldots$ We shall show its explicit form in appendix B. Note that the factor of 2 in the second and third term on the right-hand side takes into account the fact that two dark-sector particles are produced via each of the corresponding processes. Again, we have made the assumption that the annihilation rate of the darksector particles into the SM neutrinos is negligible. Such assumption is valid as long as $T_{D} \ll T$, which implies the number density of either $\chi$ or $\phi$ is much smaller than that of the SM neutrinos, and is also consistent with the second law of thermodynamics - the dark sector can never be hotter than the SM thermal bath which populates it in the first place. We shall see later that the decay and inverse decay could potentially thermalize $\phi$ with the SM neutrinos. However, this has little impact for the yield of $\chi$, if any.

While in thermal equilibrium, the dark-sector energy density is only a function of the dark-sector temperature $T_{D}$ once the masses of $\chi$ and $\phi$ are fixed. Therefore, at every step of the numerical simulation, $T_{D}$ can be obtained by solving

$$
\rho_{D}\left(T_{D}\right)=\xi_{\chi} \int \frac{d^{3} p}{(2 \pi)^{3}} \frac{E_{\chi}}{e^{E_{\chi} / T_{D}}+1}+\xi_{\phi} \int \frac{d^{3} p}{(2 \pi)^{3}} \frac{E_{\phi}}{e^{E_{\phi} / T_{D}}-1},
$$

in which $\xi_{\chi}=2$ and $\xi_{\phi}=1$ are the number of degrees of freedom for $\chi$ and $\phi$. After obtaining the time dependence of the dark sector temperature $T_{D}(t)$, one can then insert it back to eq. (3.13) and solve for $n_{\chi}$.

In figure 4, we present our benchmark results in the reannihilation regime including the evolution of the energy-transfer rates, the particle exchange rates, as well as the darkmatter yield. The upper two panels show the cases in which the dark sector undergoes a period of dark thermal equilibrium and then freezes out. By contrast, the cases with the additional QSE phase before the dark freeze-out are shown in the lower panels. Comparing with the pure freeze-in cases in figure 3, we have enhanced the coupling of $\phi$ to the dark matter, and at the same time lowered its coupling to the neutrinos in order to avoid 

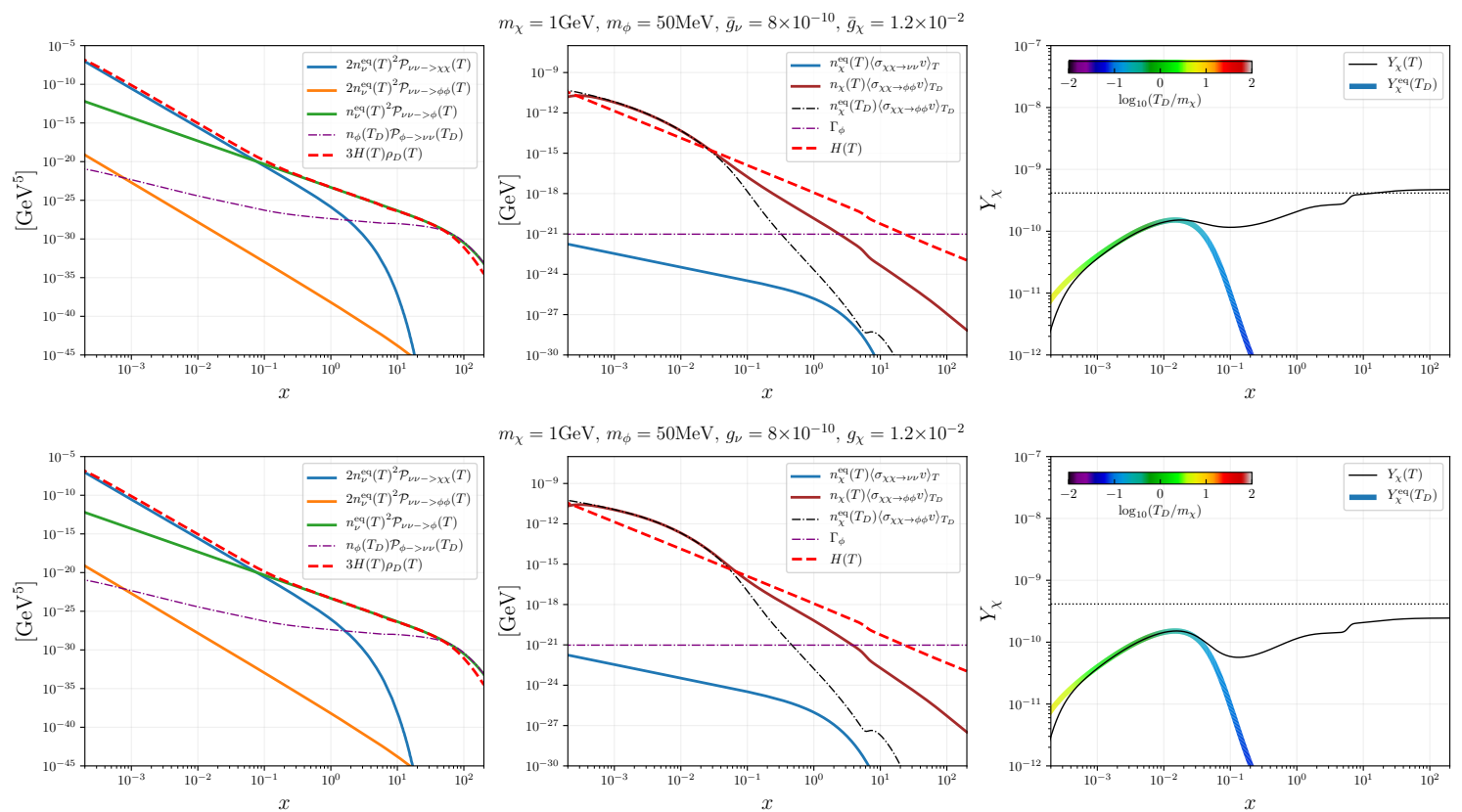

$m_{\chi}=1 \mathrm{GeV}, m_{\phi}=50 \mathrm{MeV}, g_{\nu}=8 \times 10^{-10}, g_{\chi}=1.2 \times 10^{-2}$
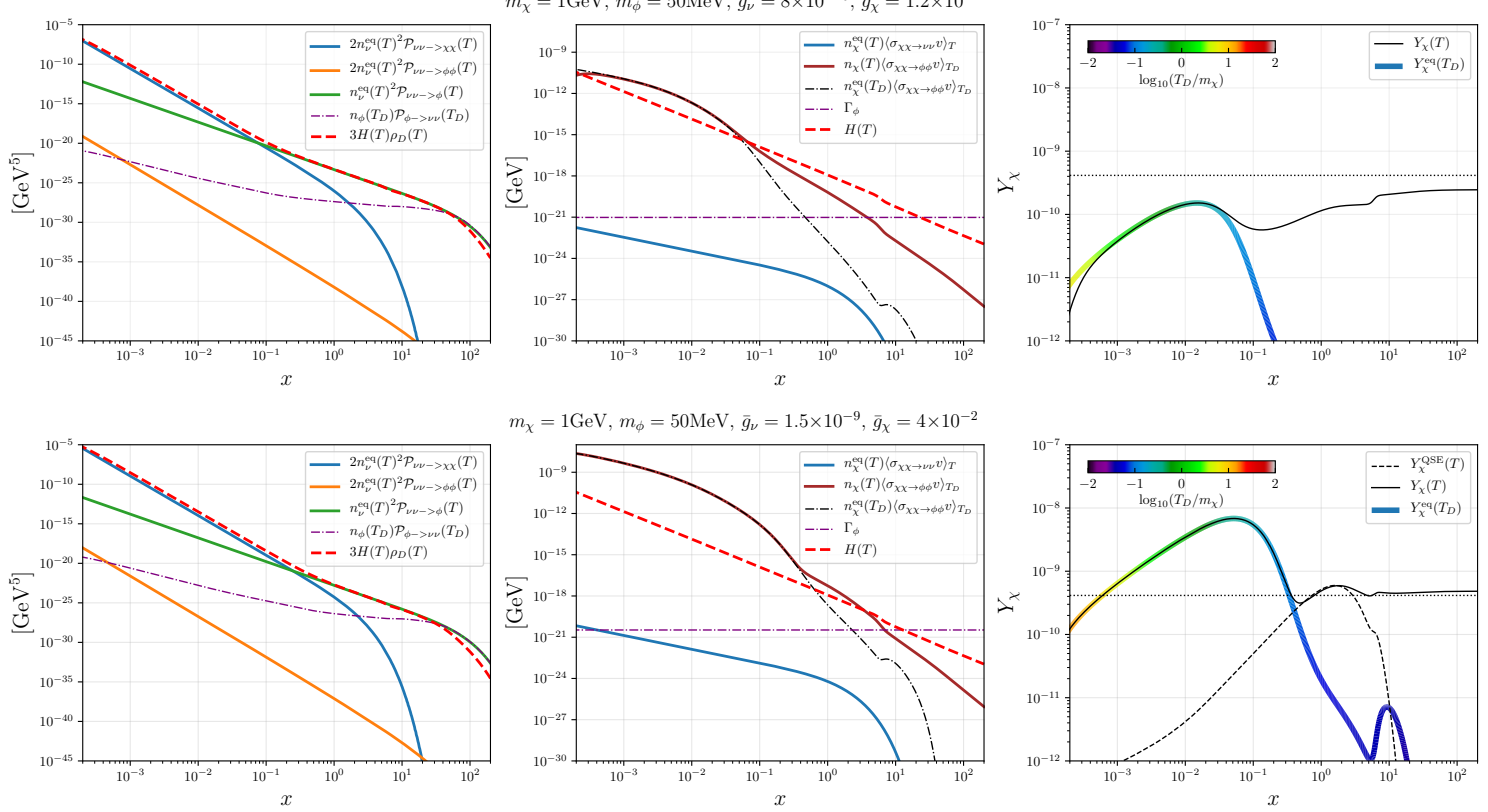

$m_{\chi}=1 \mathrm{GeV}, m_{\phi}=50 \mathrm{MeV}, \bar{g}_{\nu}=1.5 \times 10^{-9}, \bar{g}_{\chi}=4 \times 10^{-2}$
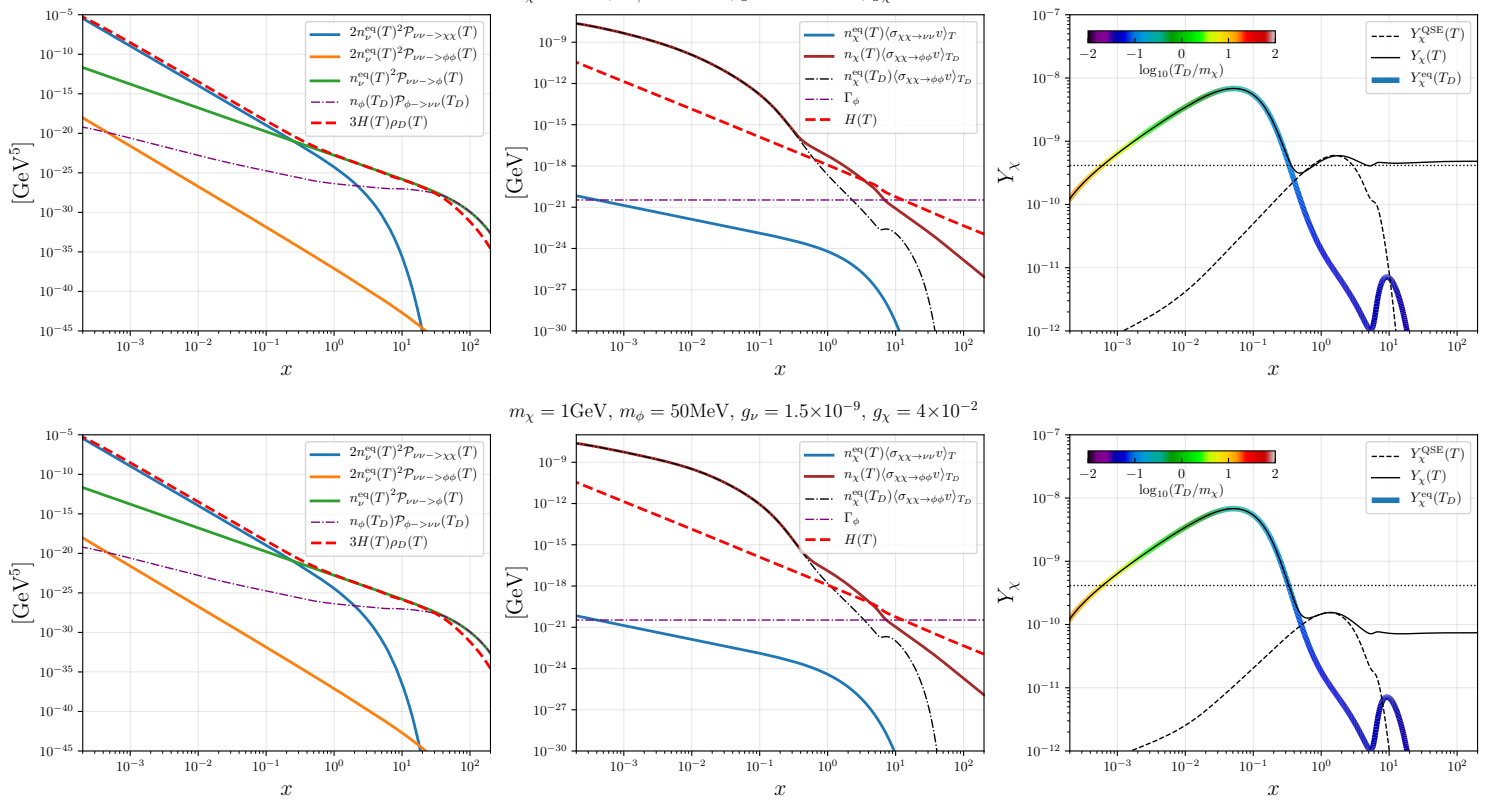

$m_{\chi}=1 \mathrm{GeV}, m_{\phi}=50 \mathrm{MeV}, g_{\nu}=1.5 \times 10^{-9}, g_{\chi}=4 \times 10^{-2}$
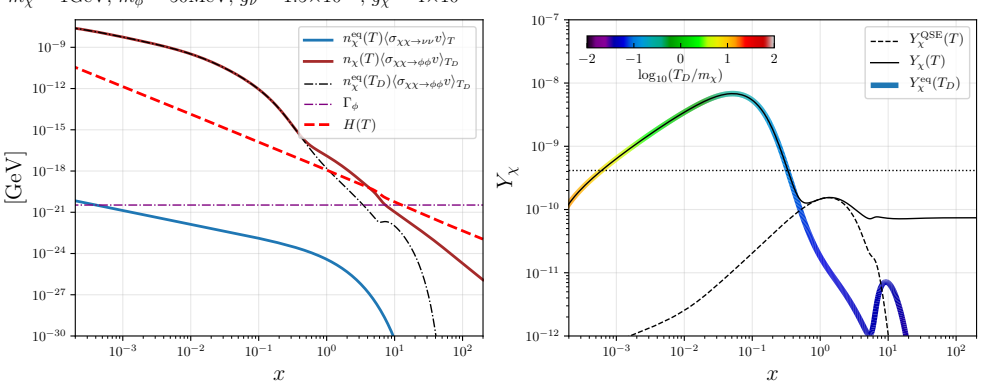

Figure 4. Benchmark results for the reannihilation regime, with the upper (lower) two panels showing the cases without (with) the QSE phase. The left columns shows the rate of the energy transfer from the SM sector as well as the rates of energy-density dilution due to the expansion of the universe. In the middle column, we compare the expansion rate with the interaction rates relevant for the dark-matter number density. In the right column, we show the evolution of $Y_{\chi}$ and $Y_{\chi}^{\mathrm{eq}}\left(T_{D}\right)$ - the yield of $\chi$ if it is in thermal equilibrium with $\phi$, which we have colored to indicate the corresponding $T_{D}$. The evolution of $\left.Y_{\chi}^{\mathrm{QSE}}(T)\right)$ is also plotted in the lower two panels for the cases with the QSE phase. 
thermalization with the SM thermal bath. The couplings are tuned such that the cases in Scenario II could have a final yield of $\chi$ similar to that of the dark matter today. At the same time, for cases with (or without) the QSE phase, we choose to use couplings of the same size just to demonstrate that the cases in Scenario I tend to have a smaller final yield due to the same reason that we have mentioned in the pure freeze-in cases.

In the left panels, we see for all the cases, most of the energy injected into the dark sector is from the $\nu \nu \rightarrow \chi \chi$ channel at early times (solid blue curve). However, the contribution from this channel decreases drastically after the SM temperature drops below the mass of the dark-matter particles, as we can tell from the quick down turn of the solid blue curve for $x \gtrsim 1$, and then the inverse decay $\nu \nu \rightarrow \phi$ (solid green curve) starts to dominate. On the other hand, the energy transfer rate of the annihilation process $\nu \nu \rightarrow \phi \phi$ (solid orange curve), is always negligibly small. These observations are consistent with the expectations from our scenario in which $\bar{g}_{\nu}$ (or $g_{\nu}$ ) is very tiny, since the amplitude of the $\nu \nu \rightarrow \phi \phi$ process is proportional to $\bar{g}_{\nu}^{2}$ (or $g_{\nu}^{2}$ ), whereas the amplitudes of the $\nu \nu \rightarrow \chi \chi$ and $\nu \nu \rightarrow \phi$ processes are proportional to $\bar{g}_{\nu} \bar{g}_{\phi}$ and $\bar{g}_{\nu}$ (or $g_{\nu} g_{\phi}$ and $g_{\nu}$ ), respectively.

Comparing the cases with and without the QSE phase, we notice that because both the coupling of $\phi$ to the dark matter and to the neutrinos are larger in the cases with the QSE phases, more energy is injected from the SM in these cases. As a result, in the middle and the right panels, while the upper two cases only reach thermal equilibrium around $x \sim 10^{-3}$, the lower two cases have already established thermal equilibrium long before that, and have acquired much more dark-matter particles when the dark sector is in thermal equilibrium. As a consequence, the evolution after reaching thermal equilibrium is also drastically different.

In the first two cases, $Y_{\chi}$ tracks $Y_{\chi}^{\mathrm{eq}}\left(T_{D}\right)$ until $n_{\chi}^{\mathrm{eq}}\left(T_{D}\right)\left\langle\sigma_{\chi \chi \rightarrow \phi \phi}\right\rangle_{T_{D}} \lesssim H$, which occurs around $x \sim 0.02-0.05$. At this time, the temperature in the dark sector $T_{D}$ is already smaller than $m_{\chi}$ as one can tell from the color variation in $Y_{\chi}^{\mathrm{eq}}\left(T_{D}\right)$. As we have mentioned above, after the dark freeze-out, the yield $Y_{\chi}$ is not fixed yet. Similar to the pure freeze-in scenario, the solid blue curve in the middle panel shows that the production of $\chi$ from the SM neutrinos is still significant until the SM temperature is much smaller than $m_{\chi}$. Therefore, with the injection from the SM thermal bath continues to contribute, the yield only approaches its asymptote after $x \gtrsim 1$.

In the bottom two cases, $Y_{\chi}$ tracks $Y_{\chi}^{\mathrm{eq}}\left(T_{D}\right)$ at the beginning, and can even grow larger than its final asymptotic value because of a larger $\bar{g}_{\nu}\left(\right.$ or $\left.g_{\nu}\right)$. These "excess" dark-matter particles are rapidly depleted by annihilating into $\phi$. As a result, as $T_{D}$ approaches and goes below $m_{\chi}, Y_{\chi}$ grows increasingly slower and eventually starts to decrease (see the green part of $\left.Y_{\chi}^{\mathrm{eq}}\left(T_{D}\right)\right)$. Note that, due to a larger coupling between $\chi$ and $\phi, Y_{\chi}$ can still track $Y_{\chi}^{\mathrm{eq}}\left(T_{D}\right)$ even when $\chi$ is already non-relativistic. As $T_{D}$ keeps dropping, the inverse process $\phi \phi \rightarrow \chi \chi$ becomes less and less important due to kinematics. However, since $\bar{g}_{\nu}$ (or $g_{\nu}$ ) is larger in these cases, the dark-matter annihilation rate $n_{\chi}\left\langle\sigma_{\chi \chi \rightarrow \phi \phi} v\right\rangle_{T_{D}}$ can still be larger than $H$. This is clearly shown in the middle panels as the solid brown curve peels off from the dash-doted black curve. Consequently, the dark matter breaks away from the thermal equilibrium and enters the QSE phase in which $Y_{\chi}$ tracks $Y_{\chi}^{\mathrm{QSE}}(T)$. Finally, when $n_{\chi}(T)\left\langle\sigma_{\chi \chi \rightarrow \phi \phi} v\right\rangle_{T_{D}} \lesssim H(T)$, the QSE can no longer be maintained. Then, $\chi$ freezes out, and the yield approaches its final value. 


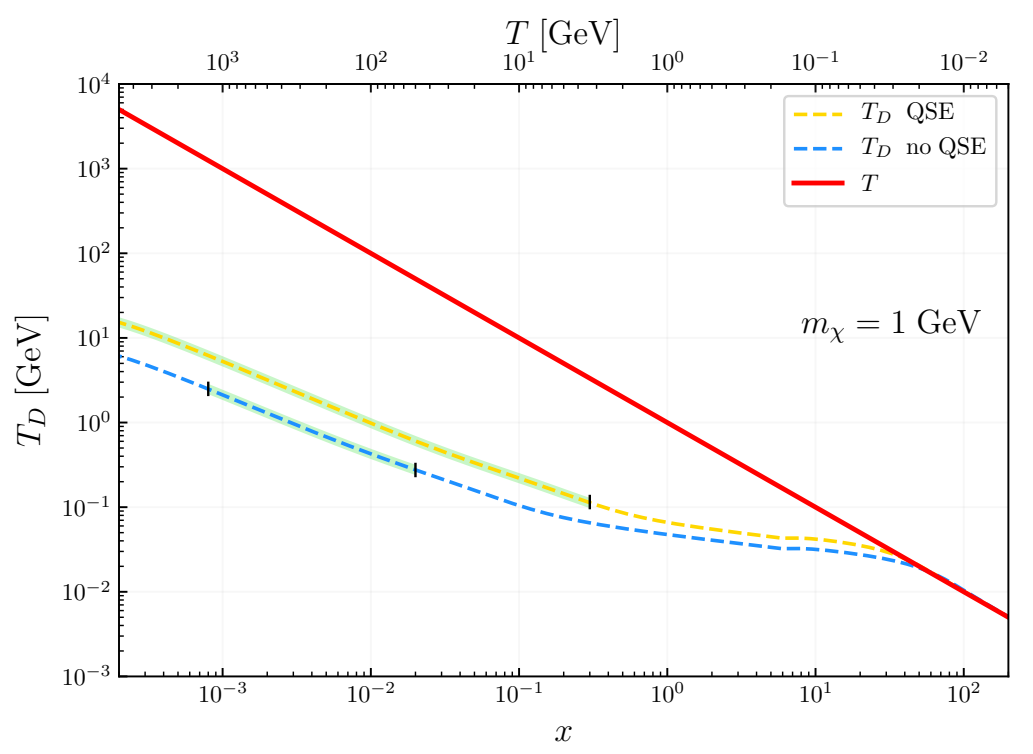

Figure 5. The evolution of $T_{D}$ as a function of $x$ (or the SM temperature $T$ ) when the dark sector is in thermal equilibrium. The yellow curve is obtained from the cases with the QSE phase, whereas the blue curve is from cases without it, and there is no visible distinction no matter $\phi$ is a scalar or pseudoscalar. Since the temperature in the dark sector is only physical when $\chi$ and $\phi$ are in thermal equilibrium, we roughly shaded the region in which the dark thermal equilibrium is established. As a reference, we use the red curve to indicate the temperature of the SM thermal bath.

\subsection{1 $T_{D}$ evolution}

We comment here on the evolution of dark-sector temperature $T_{D}$ in the reannihilation regime. The curves for $T_{D}$ which are obtained by solving eq. (3.17) are shown explicitly in figure 5 . Note that, we only include two curves for $T_{D}$ because there is no visible distinction between the scalar and pseudoscalar cases. It is worth emphasizing that, though the solution to eq. (3.17) is unique and exists in the entire regime we are considering, $T_{D}$ is only physically meaningful when the dark-sector particles $\chi$ and $\phi$ are in thermal equilibrium. We therefore shade the region in which the dark thermal equilibrium is reached. Within this region, we can see that slopes of both $T_{D}$ curves are less steep than the that of $T$. This is because while the energy density of the SM thermal bath is only subject to the expansion of the universe and some minor impact from the change of $g_{\star}$, the dark-sector energy density is in addition always sourced by the SM. Therefore, its temperature $T_{D}$ decreases slower than $T$. After $\chi$ and $\phi$ decouple, the momentum distributions of $\chi$ and $\phi$ evolve independently. As we can see from the left panels of figure 4, the inverse decay of neutrinos into $\phi$ starts to surpass the annihilation into a pair of $\chi$ around $x \sim 0.1$, which is shortly before or after $\phi$ and $\chi$ decouple. After that, most of the energy injection from the SM thermal bath goes into $\phi$. However, the energy that goes into $\phi$ and cannot be rapidly converted into $\chi$ since $\chi$ is already non-relativistic when it decouples. The energy density in the dark sector is thus dominated by $\phi$ after decoupling. Therefore, assuming that the kinetic equilibrium is still maintained for $\phi$, and that chemical potential is negligible, the 
$T_{D}$ curves to the right of the shaded region largely reflect the evolution of the energy density of $\phi$, i.e. $T_{D} \approx T_{\phi}$ since $\rho_{D} \approx \rho_{\phi}^{\text {eq }}\left(T_{D}\right)$. However, $T_{D}$ always tends to be an overestimate for $T_{\phi}$ in this region as $\rho_{\chi}>\rho_{\chi}^{\text {eq }}\left(T_{D}\right)$ after $\chi$ decouple. Eventually, when $H(T) \sim \Gamma_{\phi}, \phi$ decays rapidly into neutrinos. For the choice of $m_{\phi}$ and $\bar{g}_{\nu}$ (or $g_{\nu}$ ) in the examples, the decay occurs around $x \sim 10-20$, when $\phi$ starts to transition from relativistic to non-relativistic. Therefore, though both $T_{D}$ curves eventually merge with $T$, which indicates that $\phi$ could thermalizes with the SM sector through decay and inverse decay, its energy density is already suppressed at that time - it is only the neutrinos living on the high momentum tail of the Fermi-Dirac distribution that are able to create the $\phi$ particles, which, in turn, quickly decay and deposit its energy back to the SM thermal bath.

\section{Model phenomenologies}

In this section, we discuss general considerations on the phenomenological aspects of our model. We notice that, at early times, the existence of the dark scalar $\phi$ might contribute to a non-negligible fraction of energy density in the universe, and its decay would inject energy into the SM thermal bath. Therefore, our model could be constrained by measurements on BBN and CMB. At late times, the interaction within the dark sector could allow a large annihilation rate inside dark-matter halos which would produce a lot of neutrinos. Thus our model also receives constraints from the current observations on astrophysical neutrinos. Moreover, the self-interaction between dark-matter particles could also suppress the formation of small structures. Therefore, we also want to explore the extent to which our model could be exploited to solve the small scale structure problems. In what follows, we discuss these aspects in turn based on the general effective Lagrangian in eq. (2.3) where both the scalar and the pseudoscalar couplings are present instead of referring to any specific UV completion. One can easily apply the results to Scenario I (Scenario II) by setting the pseudoscalar (scalar) coupling to zero.

\subsection{Dark scalar decay}

In general, the presence of $\phi$ and its decay products during the epochs relevant for BBN and CMB would increase the expansion rate of the universe and could change the temperature ratio between photons and neutrinos. Such effects could eventually be constrained by the observed abundance of light elements and the CMB power spectrum. Since it is not the purpose of this paper to perform a full BBN analysis on the light element abundances, and the recent observation on $\mathrm{CMB}$ provides stringent constraint on the effective number of neutrino species with $N_{\text {eff }}=2.99 \pm 0.17$ [3] consistent with the SM prediction $N_{\text {eff }}=3.046$ [51], in this subsection, we shall base our analysis solely on $N_{\text {eff }}$.

In our model, using eq. (A.7), the SM temperature at which the decay occurs can be roughly estimated by setting $H\left(T_{\mathrm{dec}}\right)=\Gamma_{\phi}$, which leads to

$$
\begin{aligned}
T_{\mathrm{dec}} & \approx \frac{3 \sqrt{\bar{g}_{\nu}^{2}+g_{\nu}^{2}}}{4 \sqrt{2} \pi}\left(\frac{10}{g_{\star}\left(T_{\mathrm{dec}}\right)}\right)^{1 / 4}\left(\frac{m_{\phi}}{\mathrm{MeV}}\right)^{1 / 2}\left(\frac{M_{P}}{\mathrm{MeV}}\right)^{1 / 2} \mathrm{MeV} \\
& \approx 10^{10} \times \sqrt{\bar{g}_{\nu}^{2}+g_{\nu}^{2}}\left(\frac{10}{g_{\star}\left(T_{\mathrm{dec}}\right)}\right)^{1 / 4}\left(\frac{m_{\phi}}{\mathrm{MeV}}\right)^{1 / 2} \mathrm{MeV} .
\end{aligned}
$$




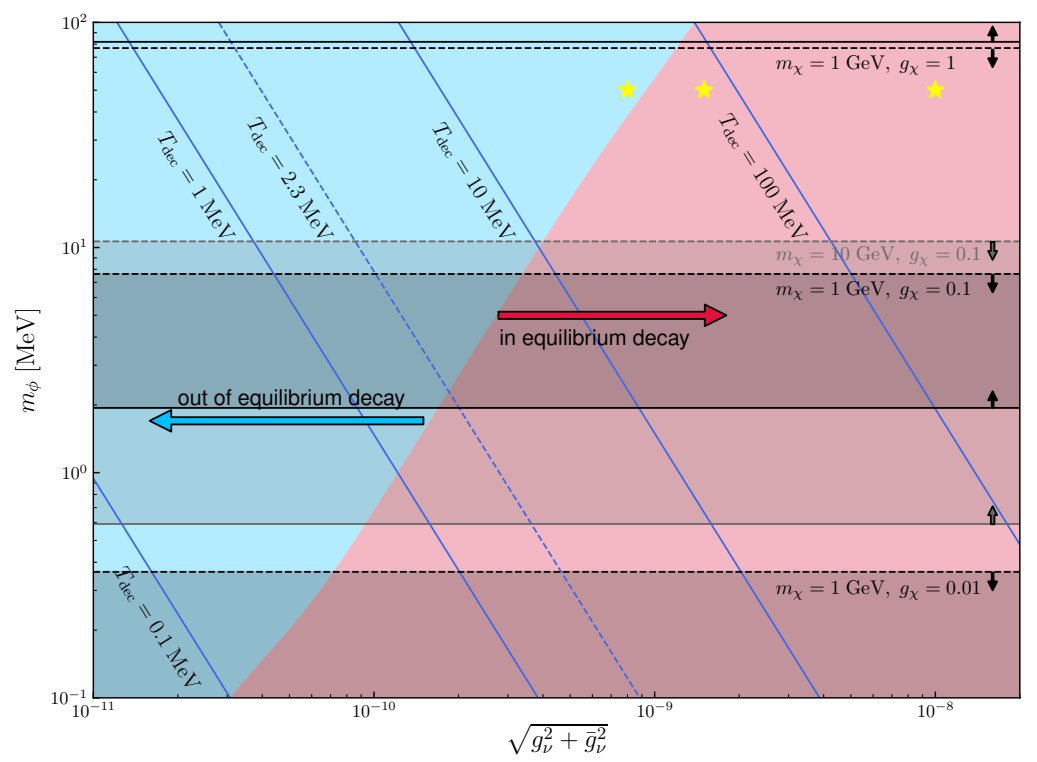

Figure 6. For different combination of $m_{\phi}$ and $\sqrt{g_{\nu}^{2}+\bar{g}_{\nu}^{2}}$, the blue contours show the values of $T_{\mathrm{dec}}$, among which the dashed blue contour indicates decaying at the temperature of neutrino decoupling. The blue and red shaded regions corresponds to out-of-equilibrium and in-equilibrium decay, respectively. The benchmarks presented in the previous section are labelled by the stars. The solid horizontal lines indicate the upper limit on $m_{\phi}$ from the Bullet Cluster constraint on self-interaction (evaluated at $v=4000 \mathrm{~km} / \mathrm{s}$ ) once $m_{\chi}$ and $g_{\chi}$ are fixed. Similarly, the dashed horizontal lines correspond to the lower limit on $m_{\phi}$ so that $\sigma_{V} / m_{\chi} \geq 1 \mathrm{~cm}^{2} / \mathrm{g}$ at $v=200 \mathrm{~km} / \mathrm{s}$ in order to solve the small scale structure problems. The shaded bands represent regions where the two conditions above are simultaneously satisfied. Note that there is no band for $m_{\chi}=1 \mathrm{GeV}, g_{\chi}=1$ as its upper bound is smaller than its lower bound. Moreover, for $m_{\chi}=1 \mathrm{GeV}, g_{\chi}=0.01$, the lower bound $m_{\phi} \simeq 0.019 \mathrm{MeV}$ is not shown within this figure.

On one hand, if $\Gamma_{\phi} / H\left(m_{\phi}\right)>1$ (or equivalently $T_{\mathrm{dec}}>m_{\phi}$ ), the decay and inverse decay would bring $\phi$ into thermal equilibrium with the SM neutrinos. On one hand, if $\Gamma_{\phi} / H\left(m_{\phi}\right)<1$ (or $T_{\mathrm{dec}}<m_{\phi}$ ), $\phi$ decays out of equilibrium. The regions for in- and out-of-equilibrium decay as well as contours for $T_{\text {dec }}$ are shown in figure 6 .

If $\phi$ decays in equilibrium, as $\phi$ becomes non-relativistic, its energy density is gradually transferred to the SM sector. Before neutrino decoupling $(T \sim 2.3 \mathrm{MeV})$, the energy transferred would not change the temperature ratio between photons and neutrinos since neutrinos are still tightly coupled with the rest of SM plasma. However, the energy transferred after the neutrino decoupling would only reheat the neutrino sector instead of the sector participating in electromagnetic interactions, and therefore give rise to an increased value of $N_{\text {eff }}$ from the SM prediction. Referring to the analysis in ref. [52] with the recent bound from Planck Collaboration [3], the existence of this additional species $\phi$ in equilibrium with the SM neutrinos leads to $m_{\phi} \gtrsim 10 \mathrm{MeV}$.

For the decay of $\phi$ out of equilibrium, similar to the previous case, if it occurs before neutrino decoupling, no observational effect would be left even as the decay products reheats the entire SM thermal bath equally. This corresponds to the blue shaded region to the 
right of the $T=2.3 \mathrm{MeV}$ contour in figure 6 , and we see the lightest possible mass for this case is $m_{\phi} \simeq 2 \mathrm{MeV}$. But if $\phi$ decays after the neutrino decoupling, it would only increase the energy density of the neutrino sector and thus lead to a positive contribution to $N_{\text {eff }}$. Such contribution depends on the energy density of $\phi$ when it is decaying. Assuming $\phi$ decays instantaneously, the current Planck constraint on $N_{\text {eff }}$ then suggests that the energy density of $\phi$ when decaying should be smaller than $\sim 10 \%$ of that of a single neutrino species. Note that, the energy density of $\phi$ depends on the entire production history, which can be calculated once the full set of parameters $\left(g_{\chi}, \bar{g}_{\chi}, g_{\nu}, \bar{g}_{\nu}, m_{\chi}\right.$ and $\left.m_{\phi}\right)$ is known.

Among the benchmarks that we have shown, the cases in figure 3 and the last two rows of figure 4 correspond to the in-equilibrium decay, whereas the cases in the first two rows of figure 4 correspond to the out-of-equilibrium decay. In the former cases, our choice of mass $m_{\phi}=50 \mathrm{MeV}$ allows $\phi$ to be already non-relativistic when BBN starts, therefore its energy density is already negligible. For the latter cases, the fact that it decays much earlier before neutrino decouples ensures that the bounds on $N_{\text {eff }}$ would not be violated. The parameter choices for these cases are labelled by the stars in figure 6 .

\subsection{Indirect detection}

The decay of $\phi$ could also be exploited in the indirect detection of dark matter. In the late-time universe, though the interaction rate between the dark matter $\chi$ the SM particle is extremely small, $\chi \chi \rightarrow \phi \phi$ followed by rapid decay of $\phi \rightarrow \nu \nu$ could potentially produce a signal that can be probed by astrophysical neutrino observation experiments. These experiments usually give bounds on the dark matter annihilation cross-section $\langle\sigma v\rangle$. In the non-relativistic regime, $\langle\sigma v\rangle$ can be expanded into powers of the relative velocity $v$ :

$$
\langle\sigma v\rangle=a+b\left\langle v^{2}\right\rangle+\mathcal{O}\left(v^{4}\right)
$$

where the angle brackets represent the average over the local dark-matter distribution at the signal source. In our model $a$ and $b$ can be expressed as:

$$
\begin{aligned}
a= & \frac{g_{\chi}^{2} \bar{g}_{\chi}^{2} m_{\chi} \sqrt{m_{\chi}^{2}-m_{\phi}^{2}}}{4 \pi\left(2 m_{\chi}^{2}-m_{\phi}^{2}\right)^{2}}, \\
b= & \frac{m_{\chi}}{24 \pi\left(2 m_{\chi}^{2}-m_{\phi}^{2}\right)^{4} \sqrt{m_{\chi}^{2}-m_{\phi}^{2}}}\left\{g_{\chi}^{4}\left(18 m_{\chi}^{6}+20 m_{\phi}^{4} m_{\chi}^{2}-34 m_{\phi}^{2} m_{\chi}^{4}-4 m_{\phi}^{6}\right)\right. \\
& \left.+\left[2 \bar{g}_{\chi}^{4}\left(m_{\chi}^{2}-m_{\phi}^{2}\right)^{3}+3 g_{\chi}^{2} \bar{g}_{\chi}^{2}\left(m_{\phi}^{6}-8 m_{\phi}^{4} m_{\chi}^{2}+20 m_{\phi}^{2} m_{\chi}^{4}-12 m_{\chi}^{6}\right)\right]\right\} .
\end{aligned}
$$

Once again, we are referring to the most general Lagrangian in eq. (2.3), and one can simply put the pseudoscalar/scalar couplings to zero for Scenario I/II.

According to the result in ref. [53], we find that the most stringent bound on the annihilation cross-section $\left\langle\sigma_{\chi \chi \rightarrow \nu \nu} v\right\rangle$ for $m_{\chi} \sim 1 \mathrm{GeV}$ comes from the Super-Kamiokande (Super-K) experiment $[54,55]$, which translates into $a \lesssim 10^{-24} \mathrm{~cm}^{3} \cdot \mathrm{s}^{-1}$ and $b \lesssim 10^{-16} \mathrm{~cm}^{3}$. $\mathrm{s}^{-1}$. We plot this constraint against $\bar{g}_{\chi}$ and $g_{\chi}$ in figure 7 where the gray region is excluded by the Super-K experiment. Note that the boundary of the gray region appears to be curved around the top left and bottom right region due to the fact that both the horizontal 


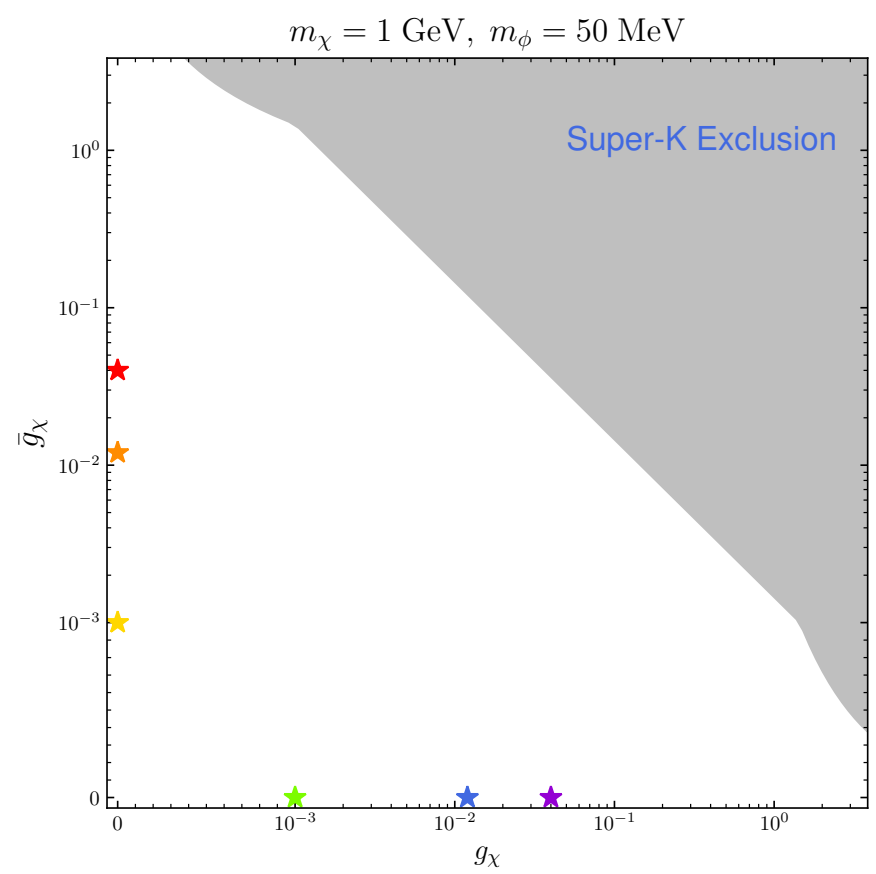

Figure 7. Indirect detection constraint from the Super-K. The excluded region is colored in gray. Different colored stars correspond to the six benchmark points in figure 3 and 4 . Note that, the axes are plotted on linear scale below $g_{\chi}, \bar{g}_{\chi}<10^{-3}$, but on $\log$ scale above it as the benchmarks all have one of the couplings $g_{\chi}$ or $\bar{g}_{\chi}$ vanished.

and vertical axes are plotted on linear scale below $10^{-3}$ but on log scale beyond it. Our benchmark points in figure 3 and 4 which all have one of the couplings $g_{\chi}$ or $\bar{g}_{\chi}$ being zero, can thus be accommodated in this figure and are labeled by the stars. In particular, along the axes, we have in the vanishing $m_{\phi}$ limit

$$
\left\{\begin{array}{ll}
\bar{g}_{\chi}<8.468 \times \sqrt{\frac{m_{\chi}}{\mathrm{GeV}}}, & \text { if } g_{\chi}=0 \\
g_{\chi}<4.889 \times \sqrt{\frac{m_{\chi}}{\mathrm{GeV}}}, & \text { if } \bar{g}_{\chi}=0
\end{array} .\right.
$$

Apparently, all our benchmarks survive the indirect detection constraint.

\subsection{Possible solution to the small scale structure problem}

Finally, we investigate the potential for our model to solve problems on small scale structures. It is well known that though the standard $\Lambda \mathrm{CDM}$ cosmology is very successful in predicting the large scale structure of the universe, it fails to predict correct structures that are compatible with the observational data at the small scales. The most prominent small-scale problems are the follows: 1) the core-cusp problems - the dark-matter density profile in the center of a galaxy predicted from simulations with collisionless cold dark matter (CDM) typically scales as $r^{-1}$ ("cuspy"), while the observations show that both cored (dark matter density $\sim r^{0}$ ) and cuspy centers exist [56]; 2) the missing satellites 
problem - the observed number of satellite galaxies in the local group is one to two orders of magnitude fewer than the prediction from CDM simulations; the too-big-to-fail problem - when matching the most luminous observed satellites of the Milky Way with the most massive subhalos from CDM simulations, the subhalos appear to be too massive to host those observed galaxies. ${ }^{2}$ These problems motivate the consideration on the self-interaction of dark-matter particles which could potentially solve the problem by lowering the density in the central region of a galaxy as compared to the CDM simulations. An excellent review on this topic is provided by the authors of ref. [40], where a rough bound on the dark-matter self-scattering cross-section over its mass $\sigma / m_{\chi}$ can be derived by fitting the astrophysical data. As pointed out in ref. [40], $\sigma / m_{\chi} \sim \mathcal{O}(1) \mathrm{cm}^{2} / \mathrm{g}$ at $v=30-200 \mathrm{~km} / \mathrm{s}$ can solve the small-scale problems, and a transition in the scaling of the velocity dependence of the self-interacting cross-section around $\mathcal{O}\left(10^{3}\right) \mathrm{km} / \mathrm{s}$ is crucial for reconciling the collisionless nature of the dark matter on large scales with the collisional behavior on small scales. As suggested by ref. [58], for the study of dark-matter self-scattering, the viscosity cross section

$$
\sigma_{V} \equiv 2 \pi \int_{0}^{\pi} \frac{d \sigma}{d \Omega}\left(1-\cos ^{2} \theta\right) \sin \theta \mathrm{d} \theta
$$

is commonly used in literature as it regulates the enhancement in forward and backward directions which is irrelevant for the thermalization of dark-matter halo, emphasizes the momentum transfer in the perpendicular direction, better describes the speed of energy equalization, and is adequate for identical particles. Therefore, we calculate $\sigma_{V}$ in our model and find that, at zero velocity limit, the relevant cross sections $\sigma_{V}^{\chi \chi \rightarrow \chi \chi}, 3$ and $\sigma_{V}^{\chi \bar{\chi} \rightarrow \chi \bar{\chi}}$ are:

$$
\begin{aligned}
\sigma_{V}^{\chi \chi \rightarrow \chi \chi} & =\frac{g_{\chi}^{4} m_{\chi}^{2}}{4 \pi m_{\phi}^{4}}, \\
\sigma_{V}^{\chi \bar{\chi} \rightarrow \chi \bar{\chi}} & =\frac{g_{\chi}^{4} m_{\chi}^{2}}{4 \pi m_{\phi}^{4}}-\frac{g_{\chi}^{2} \bar{g}_{\chi}^{2} m_{\chi}^{2}}{4 \pi m_{\phi}^{2}\left(m_{\phi}^{2}-4 m_{\chi}^{2}\right)}+\frac{\bar{g}_{\chi}^{4} m_{\chi}^{2} m_{\phi}^{4}}{2\left(m_{\phi}^{2}-4 m_{\chi}^{2}\right)^{2}} .
\end{aligned}
$$

The full forms of these two cross sections can be found in the appendix A. We define the $\sigma_{V}$ that characterizes the self-interaction strength as the summation of the above two viscosity cross sections. Notice that, in the case where $m_{\phi} \ll m_{\chi}$, its dependence on $\bar{g}_{\chi}$ are suppressed at least by $\left(m_{\phi} / m_{\chi}\right)^{2}$. Therefore

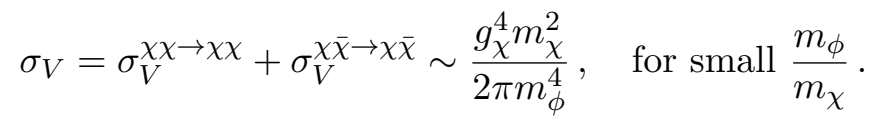

This makes it impossible for Scenario II, which only has the pseudoscalar coupling, to solve the problems on small scale structures. Our result of $\sigma_{V} / m_{\chi}$ is shown in figure 8 , where we take six benchmark points with $m_{\chi}=1,10 \mathrm{GeV}$ and $m_{\phi}=10,50 \mathrm{MeV}$ to illustrate the

\footnotetext{
${ }^{2}$ This is the too-big-to-fail problem in the Local Group. The too-big-to-fail problem in the field refers to the fact that the rotation curves for a large fraction of the dwarf galaxies indicates that they reside in halos with masses that are smaller than what is predicted using abundance matching between the observation and the CDM simulations [57] — implying that some of the more massive halos fail in forming their galactic counterpart.

${ }^{3}$ This also includes $\bar{\chi} \bar{\chi} \rightarrow \bar{\chi} \bar{\chi}$, just to simplify the notation.
} 


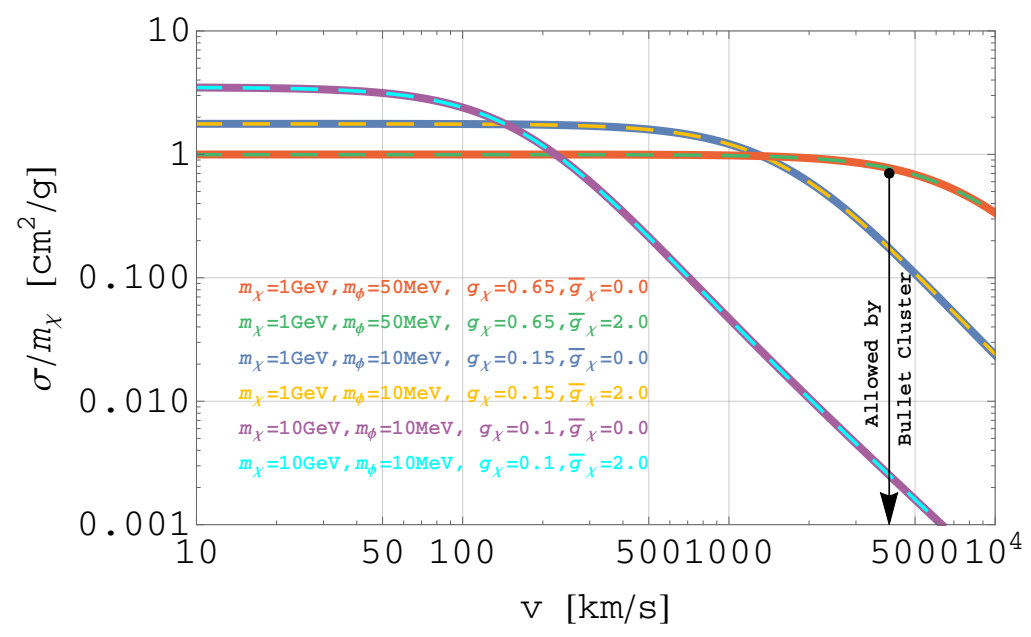

Figure 8. Viscosity cross-section $\sigma_{V}$ per dark mass $m_{\chi}$ versus the velocity with six curves showing three different combinations for $m_{\chi}=1,10 \mathrm{GeV}$ and $m_{\phi}=10,50 \mathrm{MeV}$. The values of $g_{\chi}$ are chosen such that, for $v<200 \mathrm{~km} / \mathrm{s}, \sigma_{V} / m_{\chi}$ is $\mathcal{O}(1) \mathrm{cm}^{2} / \mathrm{g}$. The values of $\bar{g}_{\chi}$ are chosen to demonstrate its negligible effect on the $\sigma_{V} / m_{\chi}$. The black arrow represents the constraint from the Bullet Cluster: $\sigma_{V} / m_{\chi} \lesssim 0.7 \mathrm{~cm}^{2} / \mathrm{g}$ for $v \sim 4000 \mathrm{~km} / \mathrm{s}[40]$.

difference in the velocity dependence. For each combination of masses, we further select a value for $g_{\chi}$ to ensure that $\sigma_{V} / m_{\chi} \sim \mathcal{O}(1) \mathrm{cm}^{2} / \mathrm{g}$ at low velocity $(v \lesssim 200 \mathrm{~km} / \mathrm{s})$ in order to solve the small scale structure problem, and two extreme values for $\bar{g}_{\chi}$ to demonstrate its negligible effect on $\sigma_{V}$.

Obviously, for the benchmark masses $\left(m_{\chi}=1 \mathrm{GeV}, m_{\phi}=50 \mathrm{MeV}\right)$ we select in figure 4 , $\mathcal{O}(1)$ value is needed for $g_{\chi}$ in order to solve the small scale problems while roughly satisfying the constraints on large scales (e.g. the Bullet Cluster constraint). However, a $g_{\chi}$ of this size would lead to a long period of dark thermal equilibrium or QSE, making the yield of dark matter too low at the dark freeze-out. In addition, since $g_{\nu}$ and/or $\bar{g}_{\nu}$ are bounded from above by the requirement on freeze-in production, increasing $g_{\nu}$ and/or $\bar{g}_{\nu}$ could not solve the problem with our choice of mass parameters. One might consider decreasing $m_{\phi}$ so that $g_{\chi}$ can be $\mathcal{O}(0.01)$, which is needed to obtain the correct relic abundance. However, for suitable values of $g_{\nu}$ and $\bar{g}_{\nu}$ that yield the correct relic abundance (roughly the same order as the values shown in figure 3 and figure 4 since it is not sensitive to $m_{\phi}$ ), and that of $g_{\chi}$ and $m_{\phi}$ that solve the small scale problems, as one can see from the darker bands in figure $6, \phi$ would have to decay in equilibrium with a mass much smaller than $10 \mathrm{MeV}$, and thus violate the $\mathrm{BBN} / \mathrm{CMB}$ constraint by presenting a sizable $\Delta N_{\text {eff }}$.

We find the only way to make our model a potentially viable SIDM candidate while satisfying the constraints on the relic abundance and BBN/CMB is to increase $m_{\chi}$. As one can see from eq. (4.9), on one hand, a larger $m_{\chi}$ could allow a smaller $g_{\chi}$ while keeping $\sigma_{V} / m_{\chi}$ large enough at low velocity. On the other hand, this would also enable $\chi$ to freeze out earlier before getting severely Boltzmann suppressed. Indeed, we find that with $m_{\chi} \sim 5-10 \mathrm{GeV}$ and $g_{\chi} \sim 0.1$, the SIDM requirement could allow $m_{\phi} \gtrsim 10 \mathrm{MeV}$. Together 


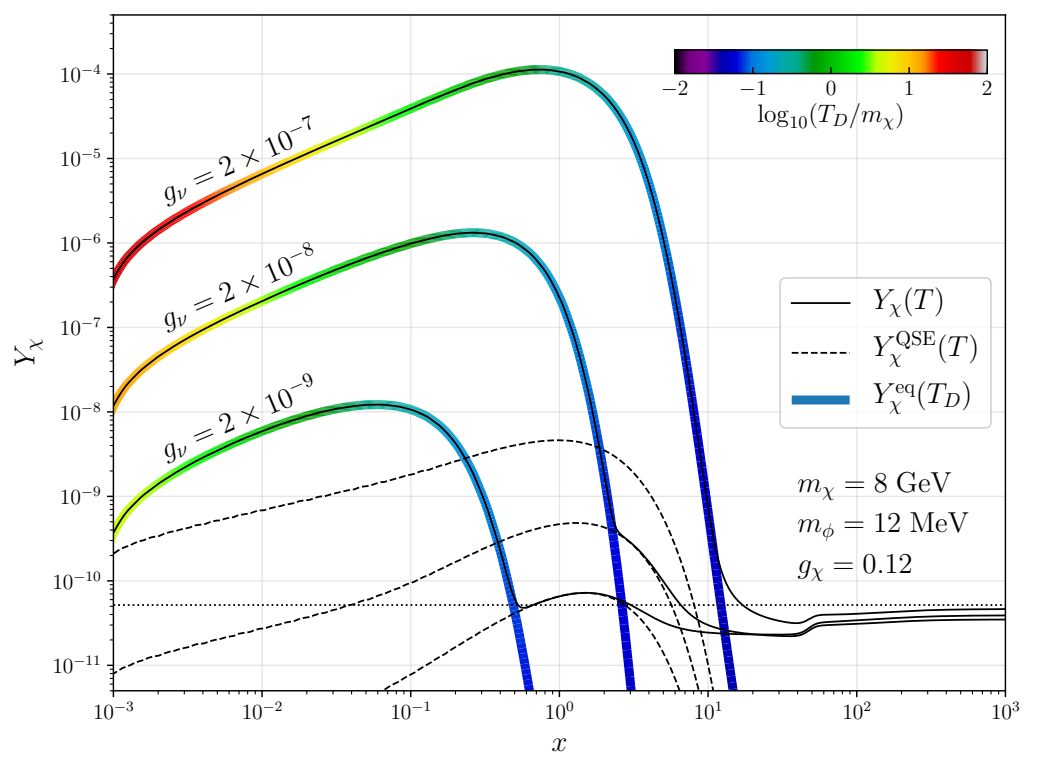

Figure 9. A few examples that satisfy the requirement from SIDM, the constraint from dark-matter decay, as well as the relic abundance. Like before, the solid black curves show the evolution of $Y_{\chi}(T)$ with the values of $g_{\nu}$ labeled alongside, the black dashed curves show $Y_{\chi}^{\mathrm{QSE}}(T)$, and the rainbowcolored curves represent $Y_{\chi}^{\mathrm{eq}}(T)$ with the colors indicating the temperature of the dark sector while in thermal equilibrium. For all the cases, the present-day relic abudance, which is indicated by the black horizontal line, is approximately satisfied. Obviously, the cases with $g_{\nu}=2 \times 10^{-9}$ and $2 \times 10^{-8}$ experience the QSE phase, whereas the case with $g_{\nu}=2 \times 10^{-7}$ shows a simple dark freeze-out without the QSE.

with $g_{\nu} \sim 10^{-9}-10^{-7}$, the constraint from BBN/CMB and the relic abundance can also be satisfied. For completeness, we show a few examples in this region of parameter space that can roughly produce the correct relic abundance in figure 9 , where we fix $m_{\chi}=8 \mathrm{GeV}$, $g_{\chi}=0.12$ and $m_{\phi}=12 \mathrm{MeV},{ }^{4}$ and plot the evolution of $Y_{\chi}$ for several different choices of $g_{\nu}$. Obviously, all the curves are in the reannihilation regime since $g_{\chi}$ is relatively large, and the cases with $g_{\nu}=2 \times 10^{-9}$ and $2 \times 10^{-8}$ exhibit the behavior of QSE.

Note that, one cannot increase $m_{\chi}$ arbitrarily. As we see from figure 8 , a larger $m_{\chi}$ would make $\sigma_{V} / m_{\chi}$ decrease faster as the velocity increases. As a result, increasing $m_{\chi}$ further beyond $\sim 10 \mathrm{GeV}$ would make $\sigma_{V} / m_{\chi}<\mathcal{O}(1) \mathrm{cm}^{2} / \mathrm{g}$ at $v \sim \mathcal{O}(100) \mathrm{km} / \mathrm{s}$, making it incompatible with the requirements for SIDM. Therefore, we find that, in the freeze-in regime, the conditions for solving the small scale structure problems (favoring larger $g_{\chi}$ and lighter $m_{\phi}$ ) tend to act against the requirement to produce the correct dark-matter relic abundance (favoring smaller $g_{\chi}$ to prevent a late dark freeze-out) and the bounds on the decay of $\phi$ (favoring $m_{\phi} \gtrsim 10 \mathrm{MeV}$ for in equilibrium decay).

As a comparison, such tension is not present in the thermal freeze-out scenario. In the thermal freeze-out scenario, the freeze-out temperature as well as the final yield can

\footnotetext{
${ }^{4}$ The range of $m_{\phi}$ that satisfy the SIDM requirement for this combination of $m_{\chi}$ and $g_{\chi}$ is $1 \lesssim m_{\phi} / \mathrm{MeV} \lesssim 14$.
} 
be estimated by

$$
\begin{aligned}
x_{f} \approx & \ln \left[0.19(n+1)\left(\xi_{\chi} / g_{\star}^{1 / 2}\right) M_{P} m_{\chi} \sigma_{0}\right] \\
& -\left(n+\frac{1}{2}\right) \ln \left\{\ln \left[0.19(n+1)\left(\xi_{\chi} / g_{\star}^{1 / 2}\right) M_{P} m_{\chi} \sigma_{0}\right]\right\}, \\
Y_{\chi}^{\text {now }} \approx & \frac{19(n+1) x_{f}^{n+1}}{\left(g_{\star, s} / g_{\star}^{1 / 2}\right) M_{P} m_{\chi} \sigma_{0}},
\end{aligned}
$$

where $\sigma_{0}=\langle\sigma v\rangle x^{n}$, with $n=0$ for $s$-wave annihilation and $n=1$ for $p$-wave annihilation. Since only the scalar coupling $g_{\chi}$ is relevant for SIDM, and it gives rise to $p$-wave annihilation, we take $n=1$ in our calculation. For $m_{\chi}=1$ and $10 \mathrm{GeV}$, we have $g_{\chi} g_{\nu} \sim 10^{-3}$ and $10^{-2}$. Thus, the tension with the relic abundance which appears in the freeze-in regime when $g_{\chi} \sim \mathcal{O}(0.1-1)$ is absent in the freeze-out regime, and it is easy to find parameter space where $\phi$ decays in equilibrium with $m_{\phi}>10 \mathrm{MeV}$.

\section{Conclusion}

In this paper, we study a simplified model describing the neutrino-portal dark matter $\chi$. In this model, the dark matter $\chi$ does not couple directly to the SM particles. Instead it interacts with the SM neutrinos via an $s$-channel exchange of the light scalar mediator $\phi$. Such neutrino-portal interaction could naturally give rise to a tiny coupling between the dark and the SM sector by relating the smallness of the coupling to the smallness of the neutrino mass, and therefore is appropriate for the freeze-in production of dark matter which requires an extremely small interaction rate between the two sectors. We then study the possible UV completions of our model and the production of dark matter in the early universe focusing on interesting dynamics that could arise in the dark sector. We also investigate several potential phenomenological constraints associated with our model. Our main results are summarized in the following:

- We point out two UV origins of the simplified model, namely, the type-I seesaw and the Majoron model, that can naturally induce a small pure scalar and pure pseudoscalar coupling between $\phi$ and neutrinos, respectively.

- We study the freeze-in production of $\chi$ in the pure freeze-in regime as well as in the reannihilation regime. For the former, we analytically estimate the relic abundance and present two benchmark results in figure 3 by solving the Boltzmann equation for the number density $n_{\chi}$ numerically. For the latter, we study the dark-sector dynamics in detail and further identify two different scenarios - the one with the dark thermal equilibrium only and the one with the additional QSE phase. Typically, the one with the QSE phase requires a larger rate of injection from the SM sector (larger $g_{\nu}$ and/or $\bar{g}_{\nu}$ ). We find that since the Boltzmann equation for the $n_{\chi}$ depends on the dark-sector temperature $T_{D}$, we need to solve the Boltzmann equation for the dark-sector energy density $\rho_{D}$ in order to obtain the evolution of $T_{D}$, and use $T_{D}$ to solve the Boltzmann equation for $n_{\chi}$. We present the other four benchmark results 
which include both scenarios for both the scalar and the pseudoscalar case. We also present and analyze the evolution of $T_{D}$ in these cases.

- We find that the decay of $\phi$ could potentially be constrained by BBN and CMB. Nevertheless, we point out that, for our benchmark results, a relatively heavy mediator with $m_{\phi} \sim 50 \mathrm{MeV}$ could avoid the BBN and CMB constraints while simultaneously give the correct relic abundance.

- The Super-K experiment is able to constrain our model by searching for dark matter annihilating to mediator $\phi$ followed by $\phi$ decaying to SM neutrinos. We show in figure 7 that our benchmarks are all allowed by the Super-K experiment.

- Since our model could allow sufficiently large self-interaction between dark-matter particles, we analyze the potential for our model to solve the small scale structure problems. We find that the self-interaction strength is sensitive to $m_{\chi}, m_{\phi}$, and to $g_{\chi}$ only. A scenario with $\bar{g}_{\chi}$ alone cannot solve small structure problems. In general, we find there is tension between the required properties for a successful SIDM candidate and the constraints on both BBN and the relic abundance in the freeze-in regime. The choice of mass parameters that we choose in the benchmarks cannot solve the problems on small scale structures. Nevertheless, it is possible to reconcile the problem by increasing the mass of $\chi$ to $\sim 5-10 \mathrm{GeV}$ while lowering $m_{\phi}$ to $\gtrsim 10 \mathrm{MeV}$. We also point out that such tension is absent in the regime of thermal freeze-out.

Finally, we would like to point out several approximations that are used in our analysis and possible extensions to our work. First of all, in our examples for UV completion, we have not considered the possibility that the heavy right-handed neutrino $N$ could also be a dark-matter candidate. Such possibility in general depends on the evolution history of the universe at high scales. We argue that, in scenarios in which the temperature of the universe has never been higher than $M_{N}$, or the mass of the inflaton is smaller than $M_{N}$, one would not expect a non-negligible population of $N$. Our analysis thus implicitly assumed an appropriate cosmological history before the scales relevant for the production of dark matter. Scenarios in which both $N$ and $\chi$ could play the role of dark matter are nevertheless worth studying.

Second, although we have confined ourselves within the freeze-in production of dark matter through the neutrino portal, if the couplings $g_{\nu}$ and/or $\bar{g}_{\nu}$ are large enough, the dark-matter particles could thermalize with the SM neutrinos and undergo thermal freezeout from the SM thermal bath. We discussed the required coupling strength for thermal freeze-out briefly by the end of section 4.3. However, we do not focus on this scenario in our paper as we are motivated by the possibility that the interactions between the dark matter and the SM particles are extremely feeble.

Third, as we have pointed out before, in the reannihilation regime, the interaction rate between the dark-sector particles depends on the dark-sector temperature $T_{D}$. However, $T_{D}$ is only physical when the $\chi$ and $\phi$ particles are in thermal equilibrium. In our analysis, we 
obtain $T_{D}$ from the energy density of the dark sector and use it to evaluate the dark-sector interaction rate throughout. Such approximation is reasonable since the interaction within the dark sector quickly becomes negligible after the dark freeze-out, and the evolution of the dark matter number density is dominated by the injection from the SM sector and the Hubble expansion.

Moreover, the $\nu-\nu-\phi$ interaction is subject to constraints from supernovae as the scalar $\phi$ with mass $\sim \mathcal{O}(10) \mathrm{MeV}$ could be produced copiously through inverse decay of SM neutrinos in the core of a supernova [59-62]. Comparing to the bound in ref. [61], part of the region favored by SIDM argument $\left(m_{\phi} \gtrsim 10 \mathrm{MeV}, g_{\nu} \sim 10^{-7}\right)$ is around the boundary of the exclusion bound from the neutrino luminosity constraint. However, the analysis there assumes a particular flavor structure for $g_{\nu}^{i j}$ and/or $\bar{g}_{\nu}^{i j}$, where only one combination of $i$ and $j$ is non-vanishing. After turning on more entries in the matrix, the exclusion bound is expected to move away to lower $g_{\nu}$ and thus allow more room for our model. Besides, the constraint from SN1987A is subject to large uncertainties. Future observation on supernova explosion would tighten or relax the constraint on our model and help identify regions of the parameter space interesting for different purposes such as the reannihilation, indirect detection and SIDM, etc. .

Furthermore, when calculating the thermally averaged cross-sections and the energy transfer rates, we have assumed that the dark-sector particles $\chi$ and $\phi$ all follow a thermal distribution. While this is true when the dark sector is in thermal equilibrium, it might not be true if the dark sector is not, as the self-scattering rate might be too small to reach kinetic equilibrium. Therefore, it is possible for the phase-space distributions of $\chi$ and $\phi$ to be highly nonthermal as the SM thermal bath keeps injecting particles into the dark sector while the existing dark-sector particles are constantly redshifting. This would in principle affect the calculation of the collision terms redas it involves integrations over the phasespace distribution. The nonthermal features in the phase-space distribution could also leave observable imprints on the matter power spectrum [63-65] if some of the $\chi$ particles could have non-negligible momenta during the structure formation. A thorough study on these effects would require solving the Boltzmann equations at the level of the phase-space distribution for both $\chi$ and $\phi$ which we leave for future work.

\section{Acknowledgments}

We would like to thank J. Heeck for useful discussions. YD acknowledges support from U.S. Department of Energy under contract No. DE-SC0011095. FH is supported by the National Natural Science Foundation of China (NSFC) under grant No. 11947302, No. 11690022, No. 11851302, No. 11675243 and No. 11761141011 and also supported by the Strategic Priority Research Program of the Chinese Academy of Sciences under grant No. XDB21010200 and No. XDB23000000. J.H.Y. is supported by the National Science Foundation of China under Grants No. 11875003 and No. 11947302. H.L.L. is supported by the

National Science Foundation of China under Grants No. 11875003 and 2019 International Postdoctoral Exchange Fellowship Program. 


\section{A Cross sections and decay width}

In this section, we present relevant cross-sections and decay widths that we have used in this paper based on the general effective Lagrangian in eq. (2.3). For all the practical purposes, we shall treat the SM neutrinos as massless.

- $\chi \chi \rightarrow \nu \nu$

$$
\sigma_{\chi \chi \rightarrow \nu \nu}=\frac{3\left(\bar{g}_{\nu}^{2}+g_{\nu}^{2}\right) s^{1 / 2}\left[g_{\chi}^{2}\left(s-4 m_{\chi}^{2}\right)+\bar{g}_{\chi}^{2} s\right]}{32 \pi \sqrt{s-4 m_{\chi}^{2}}\left[\left(s-m_{\phi}^{2}\right)^{2}+m_{\phi}^{2} \Gamma_{\phi}^{2}\right]} .
$$

- $\phi \phi \rightarrow \nu \nu$

$$
\sigma_{\phi \phi \rightarrow \nu \nu}=\frac{3\left(\bar{g}_{\nu}^{2}+g_{\nu}^{2}\right)^{2}}{8 \pi s\left(s-4 m_{\phi}^{2}\right)}\left\{\frac{\left(s^{2}-4 m_{\phi}^{2} s+2 m_{\phi}^{4}\right)}{s-2 m_{\phi}^{2}} \ln A-3 \sqrt{s\left(s-4 m_{\phi}^{2}\right)}\right\},
$$

where we have defined

$$
A \equiv \frac{s-2 m_{\phi}^{2}+\sqrt{s\left(s-4 m_{\phi}^{2}\right)}}{s-2 m_{\phi}^{2}-\sqrt{s\left(s-4 m_{\phi}^{2}\right)}}
$$

in order to simplify the expression.

- $\chi \chi \rightarrow \phi \phi$

$$
\begin{aligned}
\sigma_{\chi \chi \rightarrow \phi \phi}= & \frac{1}{32 \pi s\left(s-4 m_{\chi}^{2}\right)} \\
& \times\left\{\frac { \operatorname { l n } B } { s - 2 m _ { \phi } ^ { 2 } } \left[\left(g_{\chi}^{2}+\bar{g}_{\chi}^{2}\right)^{2} s^{2}-4\left(g_{\chi}^{2}+\bar{g}_{\chi}^{2}\right)\left(\left(g_{\chi}^{2}+\bar{g}_{\chi}^{2}\right) m_{\phi}^{2}-4 g_{\chi}^{2} m_{\chi}^{2}\right) s\right.\right. \\
& \left.+6\left(g_{\chi}^{2}+\bar{g}_{\chi}^{2}\right)^{2} m_{\phi}^{4}-16 g_{\chi}^{2}\left(g_{\chi}^{2}+\bar{g}_{\chi}^{2}\right) m_{\phi}^{2} m_{\chi}^{2}-32 g_{\chi}^{4} m_{\chi}^{2}\right] \\
& -\frac{\sqrt{\left(s-4 m_{\chi}^{2}\right)\left(s-4 m_{\phi}^{2}\right)}}{m_{\chi}^{2} s-4 m_{\phi}^{2} m_{\chi}^{2}+m_{\phi}^{4}}\left[g_{\chi}^{4}\left(2 m_{\chi}^{2}\left(s+8 m_{\chi}^{2}\right)-16 m_{\phi}^{2} m_{\chi}^{2}+3 m_{\phi}^{4}\right)\right. \\
& \left.\left.+\bar{g}_{\chi}^{4}\left(2 m_{\chi}^{2} s-8 m_{\phi}^{2} m_{\chi}^{2}+3 m_{\phi}^{4}\right)+2 g_{\chi}^{2} \bar{g}_{\chi}^{2}\left(2 m_{\chi}^{2} s-12 m_{\phi}^{2} m_{\chi}^{2}+3 m_{\phi}^{4}\right)\right]\right\},
\end{aligned}
$$

where

$$
B \equiv \frac{s-2 m_{\phi}^{2}+\sqrt{\left(s-4 m_{\chi}^{2}\right)\left(s-4 m_{\phi}^{2}\right)}}{s-2 m_{\phi}^{2}-\sqrt{\left(s-4 m_{\chi}\right)\left(s-4 m_{\phi}^{2}\right)}} .
$$

- $\nu \nu \rightarrow \phi$

$$
\sigma_{\nu \nu \rightarrow \phi}=\frac{3 \pi\left(g_{\nu}^{2}+\bar{g}_{\nu}^{2}\right) m_{\phi}^{2}}{4 s^{3 / 2}} \delta\left(\sqrt{s}-m_{\phi}\right)
$$

- $\phi \rightarrow \nu \nu$

$$
\Gamma_{\phi \rightarrow \nu \nu}=\frac{3\left(g_{\nu}^{2}+\bar{g}_{\nu}^{2}\right) m_{\phi}}{32 \pi}
$$


Viscosity cross sections for dark matter self-interaction:

- $\chi \chi \rightarrow \chi \chi$

$$
\begin{aligned}
\sigma_{V}^{\chi \chi} & \rightarrow \chi \chi=\frac{1}{96 \pi s\left(s-4 m_{\chi}^{2}\right)^{3}\left(s-4 m_{\chi}^{2}+2 m_{\phi}^{2}\right)} \\
\times & \left\{2 ( 4 m _ { \chi } ^ { 2 } - s ) ( s - 4 m _ { \chi } ^ { 2 } + 2 m _ { \phi } ^ { 2 } ) \left[g_{\chi}^{4}\left(560 m_{\chi}^{4}-64 m_{\chi}^{2} s+42 m_{\phi}^{4}+6 m_{\phi}^{2}\left(s-52 m_{\chi}^{2}\right)-s^{2}\right)\right.\right. \\
& +2 g_{\chi}^{2} \bar{g}_{\chi}^{2}\left(64 m_{\chi}^{4}-20 m_{\chi}^{2} s+30 m_{\phi}^{4}-6 m_{\phi}^{2}\left(20 m_{\chi}^{2}+s\right)+s^{2}\right) \\
& \left.+\bar{g}_{\chi}^{4}\left(-\left(s-4 m_{\chi}^{2}\right)^{2}+42 m_{\phi}^{4}+6 m_{\phi}^{2}\left(s-4 m_{\chi}^{2}\right)\right)\right] \\
& -24 \ln \left(\frac{m_{\phi}^{2}}{s-4 m_{\chi}^{2}+m_{\phi}^{2}}\right)\left[g _ { \chi } ^ { 4 } \left(16 m_{\chi}^{4}\left(s-4 m_{\chi}^{2}\right)^{2}+7 m_{\phi}^{8}+m_{\phi}^{6}\left(8 s-80 m_{\chi}^{2}\right)\right.\right. \\
& \left.+2 m_{\phi}^{4}\left(160 m_{\chi}^{4}-38 m_{\chi}^{2} s+s^{2}\right)-4 m_{\phi}^{2}\left(128 m_{\chi}^{6}-52 m_{\chi}^{4} s+5 m_{\chi}^{2} s^{2}\right)\right) \\
& +2 g_{\chi}^{2} \bar{g}_{\chi}^{2} m_{\phi}^{2}\left(-6 m_{\chi}^{2}\left(s-4 m_{\chi}^{2}\right)^{2}+5 m_{\phi}^{6}+m_{\phi}^{4}\left(4 s-40 m_{\chi}^{2}\right)+26 m_{\phi}^{2} m_{\chi}^{2}\left(4 m_{\chi}^{2}-s\right)\right) \\
& \left.\left.+\bar{g}_{\chi}^{4} m_{\phi}^{4}\left(2\left(s-4 m_{\chi}^{2}\right)^{2}+7 m_{\phi}^{4}+8 m_{\phi}^{2}\left(s-4 m_{\chi}^{2}\right)\right)\right]\right\} .
\end{aligned}
$$

- $\chi \bar{\chi} \rightarrow \chi \bar{\chi}$

$$
\begin{aligned}
\sigma_{V}^{\chi \bar{\chi}} \rightarrow \chi \bar{\chi} & =\frac{1}{32 s}\left\{\frac{2\left(g_{\chi}^{2}\left(s-4 m_{\chi}^{2}\right)+\bar{g}_{\chi}^{2} s\right)^{2}}{\left(m_{\phi}^{2}-s\right)^{2}}-\frac{2\left(g_{\chi}^{2}+\bar{g}_{\chi}^{2}\right)\left(4 m_{\chi}^{2}-s\right)\left(g_{\chi}^{2}\left(4 m_{\chi}^{2}+s\right)+\bar{g}_{\chi}^{2} s\right)}{\left(s-4 m_{\chi}^{2}\right)\left(m_{\phi}^{2}-s\right)}\right. \\
& +\frac{32 g_{\chi}^{4}+2\left(g_{\chi}^{2}+\bar{g}_{\chi}^{2}\right)^{2}\left(2 m_{\phi}^{4}+m_{\phi}^{2} s\right)-8 m_{\chi}^{2} m_{\phi}^{2}\left(g_{\chi}^{2}+\bar{g}_{\chi}^{2}\right)\left(3 g_{\chi}^{2}+\bar{g}_{\chi}^{2}\right)}{m_{\phi}^{2}\left(s-4 m_{\chi}^{2}+m_{\phi}^{2}\right)} \\
& +2 \ln \left(\frac{m_{\phi}^{2}}{s-4 m_{\chi}^{2}+m_{\phi}^{2}}\right)\left[\frac{g_{\chi}^{4}\left(4 m_{\chi}^{2}\left(s-4 m_{\chi}^{2}\right)+m_{\phi}^{2}\left(4 m_{\phi}^{2}+s\right)\right)}{\left(s-4 m_{\chi}^{2}\right)\left(m_{\phi}^{2}-s\right)}\right. \\
& \left.\left.+\frac{2 g_{\chi}^{2} \bar{g}_{\chi}^{2}\left(2 m_{\chi}^{2} s+m_{\phi}^{2}\left(2 m_{\chi}^{2}+s\right)\right)+\bar{g}_{\chi}^{4} m_{\phi}^{2} s}{\left(s-4 m_{\chi}^{2}\right)\left(m_{\phi}^{2}-s\right)}+\frac{4\left(g_{\chi}^{2}+\bar{g}_{\chi}^{2}\right)^{2} m_{\phi}^{2}-4 g_{\chi}^{2}\left(g_{\chi}^{2}+\bar{g}_{\chi}^{2}\right) m_{\chi}^{2}}{s-4 m_{\chi}^{2}}\right]\right\} .
\end{aligned}
$$

\section{B Energy transfer}

In this appendix, we present the explicit forms for the energy transfer terms in eq. (3.16). Let us begin with the Boltzmann equation for the phase-space distribution of a particle species $\psi$ :

$$
\frac{\partial f_{\psi}\left(p_{\psi}, t\right)}{\partial t}=H(t) p_{\psi} \frac{\partial f_{\psi}\left(p_{\psi}, t\right)}{\partial p_{\psi}}+C[f],
$$

in which $C[f]$ is the collision operator which involves all possible processes for $\psi$, e.g. decay, inverse decay, elastic scattering, annihilation, etc., and thus depends on the phase-space 
distribution of all relevant species. For a specific process $\psi+a+b+\cdots \leftrightarrow i+j+\ldots$, the collision term takes the general form

$$
\begin{aligned}
C[f]=- & \frac{1}{2 E_{\psi}} \int d \pi_{a} d \pi_{b} \ldots d \pi_{i} d \pi_{j} \ldots(2 \pi)^{4} \delta^{(4)}\left(p_{\psi}+p_{a}+p_{b}+\ldots-p_{i}-p_{j}-\ldots\right) \\
\times & {\left[\left|\mathcal{M}_{\psi+a+b+\cdots \leftrightarrow i+j+\ldots}\right|^{2} f_{\psi} f_{a} f_{b} \ldots\left(1 \pm f_{i}\right)\left(1 \pm f_{j}\right) \ldots\right.} \\
& \left.\quad-\left|\mathcal{M}_{i+j+\cdots \leftrightarrow \psi+a+b+\ldots}\right|^{2} f_{i} f_{j} \ldots\left(1 \pm f_{\psi}\right)\left(1 \pm f_{a}\right)\left(1 \pm f_{b}\right) \ldots\right]
\end{aligned}
$$

where $d \pi_{i}=d^{3} p_{i} /\left((2 \pi)^{3} 2 E_{i}\right)$, and " \pm " represents the Bose-enhancement/Pauli-blocking effects - one needs to choose "+" for bosons whereas "-" for fermions. In principle, the phase-space distributions $f$ could take any form. For species in thermal equilibrium, $f$ is either Bose-Einstein or Fermi-Dirac. However, for most of the practical purposes, the Maxwell-Boltzmann distribution can be used as a good approximation, and the condition $f \ll 1$ is often satisfied. Therefore, one can use the approximations $f \approx e^{-E / T}$ and $1 \pm f \approx 1$ which greatly simplify the calculation.

To get the energy-transfer terms in the Boltzmann equation for the energy density, we just need to multiply eq. (B.1) with $E_{\psi}$ and integrate over the phase-space element $d^{3} p_{\psi} /(2 \pi)^{3}$. The result is

$$
\frac{\partial \rho_{\psi}}{\partial t}=-3 H\left(P_{\psi}+\rho_{\psi}\right)+\int \frac{d^{3} p_{\psi}}{(2 \pi)^{3}} E_{\psi} C[f]
$$

For the process $\psi+a+b+\cdots \leftrightarrow i+j+\ldots$, one can separate the contribution from the forward and backward directions, i.e. the contribution from the two terms in the square brackets in eq. (B.2). Thus, the last term can be written formally as

$$
\int \frac{d^{3} p_{\psi}}{(2 \pi)^{3}} E_{\psi} C[f]=-n_{\psi} n_{a} n_{b} \ldots \mathcal{P}_{\psi+a+b+\cdots \rightarrow i+j+\ldots}+n_{i} n_{j} \ldots \mathcal{P}_{i+j+\cdots \rightarrow \psi+a+b+\ldots}
$$

In the following subsections, we shall calculate the energy transfer terms for several particular processes.

- Decay: $\phi \rightarrow \nu \nu$

The contribution from the decay process $\phi \rightarrow \nu \nu$ is

$$
\begin{aligned}
n_{\phi} \mathcal{P}_{\phi \rightarrow \nu \nu} & =\int d \pi_{\phi} f_{\phi} E_{\phi} \int d \pi_{\nu_{1}} \int d \pi_{\nu_{2}}\left|\mathcal{M}_{\phi \rightarrow \nu \nu}\right|^{2}(2 \pi)^{4} \delta^{(4)}\left(p_{\nu_{1}}+p_{\nu_{2}}-p_{\phi}\right) \\
& =\int \frac{d^{3} p_{\phi}}{(2 \pi)^{3}} f_{\phi} m_{\phi} \frac{1}{2 m_{\phi}} \int d \pi_{\nu_{1}} \int d \pi_{\nu_{2}}\left|\mathcal{M}_{\phi \rightarrow \nu \nu}\right|^{2}(2 \pi)^{4} \delta^{(4)}\left(p_{\nu_{1}}+p_{\nu_{2}}-p_{\phi}\right) \\
& =n_{\phi} m_{\phi} \Gamma_{\phi \rightarrow \nu \nu}
\end{aligned}
$$

where $\Gamma_{\phi \rightarrow \nu \nu}$ is the decay width in the center of mass frame of $\phi$. 
- Inverse Decay: $\nu \nu \rightarrow \phi$

The energy-transfer term of this process is

$$
n_{\nu}^{\mathrm{eq}}(T)^{2} \mathcal{P}_{\nu \nu \rightarrow \phi}=\int \frac{d^{3} p_{\nu_{1}}}{(2 \pi)^{3}} \frac{d^{3} p_{\nu_{2}}}{(2 \pi)^{3}} \sigma_{\nu \nu \rightarrow \phi} v_{\mathrm{M} \varnothing \mathrm{l}}\left(E_{\nu_{1}}+E_{\nu_{2}}\right) e^{-E_{\nu_{1}} / T} e^{-E_{\nu_{2}} / T}
$$

Here we use the superscript "eq" to emphasize that neutrinos are in thermal equilibrium with the rest of the SM thermal bath. Following the convention in Gondolo's paper:

$$
\begin{aligned}
E_{+} & =E_{\nu_{1}}+E_{\nu_{2}}, \\
E_{-} & =E_{\nu_{1}}-E_{\nu_{2}}, \\
E_{-}^{\min } & =-\sqrt{1-4 m_{\nu}^{2} / s} \sqrt{E_{+}^{2}-s}, \\
E_{-}^{\max } & =\sqrt{1-4 m_{\nu}^{2} / s} \sqrt{E_{+}^{2}-s}, \\
v_{\mathrm{M} \varnothing 1} E_{\nu_{1}} E_{\nu_{2}} & =\frac{1}{2} \sqrt{s\left(s-4 m_{\nu}^{2}\right)},
\end{aligned}
$$

the above integration becomes

$$
\begin{aligned}
& \frac{1}{32 \pi^{4}} \int_{4 m_{\nu}^{2}}^{\infty} d s \int_{\sqrt{s}}^{\infty} d E_{+} \int_{E_{-}^{\min }}^{E_{-}^{\max }} d E_{-} \sigma_{\nu \nu \rightarrow \phi} v_{\mathrm{M} \varnothing \mathrm{l}} E_{\nu_{1}} E_{\nu_{2}} E_{+} e^{-E_{+} / T} \\
& =\frac{1}{32 \pi^{4}} \int_{4 m_{\nu}^{2}}^{\infty} d s \sigma_{\nu \nu \rightarrow \phi}\left(s-4 m_{\nu}^{2}\right) \int_{\sqrt{s}}^{\infty} d E_{+} \sqrt{E_{+}^{2}-s} E_{+} e^{-E_{+} / T} .
\end{aligned}
$$

Let us first integrate over $d E_{+}$

$$
\int_{\sqrt{s}}^{\infty} d E_{+} E_{+} \sqrt{E_{+}^{2}-s} e^{-E_{+} / T}=s T K_{2}(\sqrt{s} / T) .
$$

Insert the result above, we obtain the final expression

$$
n_{\nu}^{\mathrm{eq}}(T)^{2} \mathcal{P}_{\nu \nu \rightarrow \phi}=\frac{1}{32 \pi^{4}} \int_{4 m_{\nu}^{2}}^{\infty} d s \sigma_{\nu \nu \rightarrow \phi} s\left(s-4 m_{\nu}^{2}\right) T K_{2}(\sqrt{s} / T) .
$$

Notice that this would be exactly the same with $n_{\phi}\left(T_{\phi}\right) \mathcal{P}_{\phi \rightarrow \nu \nu}$ in eq. (B.5) if $\phi$ is in thermal equilibrium with the SM thermal bath.

- 2-to-2 process

Let us consider the 2 -to- 2 process $3+4 \leftrightarrow 1+2$. The energy-transfer collision term associated with this process for particle 1 is

$$
\begin{aligned}
n_{3} n_{4} \mathcal{P}_{34 \rightarrow 12}-n_{1} n_{2} \mathcal{P}_{12 \rightarrow 34}= & \int \prod_{i=1}^{4} d \pi_{i}(2 \pi)^{4} \delta^{(4)}\left(p_{1}+p_{2}-p_{3}+p_{4}\right) \\
& \times E_{1}\left[f_{3}\left(p_{3}\right) f_{4}\left(p_{4}\right) \overline{\left|\mathcal{M}_{34 \rightarrow 12}\right|^{2}}-f_{1}\left(p_{1}\right) f_{2}\left(p_{2}\right) \overline{\left|\mathcal{M}_{12 \rightarrow 34}\right|^{2}}\right],
\end{aligned}
$$

where the "bar" over the amplitude $|\mathcal{M}|^{2}$ indicates necessary average over different spin states. For our purpose, we assume that particles 3 and 4 are the states in the SM thermal 
bath, whereas particles 1 and 2 are dark-sector states. Since for freeze-in process, the energy transferred from the dark sector to the visible sector is negligible, we just need to calculate the energy transferred to the dark sector through the above process. Therefore, we just need to calculate the forward process - the part associated with the first term in the brackets:

$$
n_{3}^{\mathrm{eq}}(T) n_{4}^{\mathrm{eq}}(T) \mathcal{P}_{34 \rightarrow 12}=\int \prod_{i=1}^{4} d \pi_{i}(2 \pi)^{4} \delta^{(4)}\left(p_{1}+p_{2}-p_{3}+p_{4}\right) \overline{\left|\mathcal{M}_{34 \rightarrow 12}\right|^{2}} E_{1} f_{3}^{\mathrm{eq}}\left(p_{3}\right) f_{4}^{\mathrm{eq}}\left(p_{4}\right) \text {. }
$$

Using detailed balance $f_{3}^{\mathrm{eq}}\left(p_{3}\right) f_{4}^{\mathrm{eq}}\left(p_{4}\right)=f_{1}^{\mathrm{eq}}\left(p_{1}\right) f_{2}^{\mathrm{eq}}\left(p_{2}\right)$ and unitarity, and inserting the flux factor $F \equiv\left[\left(p_{1} \cdot p_{2}\right)^{2}-m_{1}^{2} m_{2}^{2}\right]^{1 / 2}[66]$, we can rewrite the above integral as

$$
\begin{aligned}
& \int \frac{d^{3} p_{1}}{(2 \pi)^{3}} \frac{d^{3} p_{2}}{(2 \pi)^{3}} f_{1}^{\mathrm{eq}}\left(p_{1}\right) f_{2}^{\mathrm{eq}}\left(p_{2}\right) E_{1} \frac{F}{E_{1} E_{2}} \\
& \quad \times \frac{1}{4 F} \int \frac{d^{3} p_{3}}{(2 \pi)^{3} 2 E_{3}} \frac{d^{3} p_{4}}{(2 \pi)^{3} 2 E_{4}}(2 \pi)^{4} \delta^{(4)}\left(p_{1}+p_{2}-p_{3}+p_{4}\right) \overline{\left|\mathcal{M}_{12 \rightarrow 34}\right|^{2}} \\
& =\int \frac{d^{3} p_{1}}{(2 \pi)^{3}} \frac{d^{3} p_{2}}{(2 \pi)^{3}} f_{1}^{\mathrm{eq}}\left(p_{1}\right) f_{2}^{\mathrm{eq}}\left(p_{2}\right) E_{1} \sigma_{12 \rightarrow 34} v_{\mathrm{M} \varnothing \mathrm{l}},
\end{aligned}
$$

in which we have used the fact that $v_{\mathrm{M} ø \mathrm{l}}=F / E_{1} E_{2}$, and the second line is simply $\sigma_{12 \rightarrow 34}$. Comparing with the second term in the square brackets of eq. (B.15), it is easy to notice that the calculation above is exactly equivalent to calculating the energy transfer rate of the inverse process $1+2 \rightarrow 3+4$ if particle 1 and 2 are in thermal equilibrium with the visible sector. This observation proves that

$$
n_{3}^{\mathrm{eq}}(T) n_{4}^{\mathrm{eq}}(T) \mathcal{P}_{34 \rightarrow 12}=n_{1}^{\mathrm{eq}}(T) n_{2}^{\mathrm{eq}}(T) \mathcal{P}_{12 \rightarrow 34},
$$

which allows us to trade the energy-transfer term of one process for that of its inverse. Following the procedure in ref. [8], the eq. (B.17) can be further simplified, and we arrive at this final expression:

$$
n_{3}^{\mathrm{eq}}(T) n_{4}^{\mathrm{eq}}(T) \mathcal{P}_{34 \rightarrow 12}=\frac{T}{64 \pi^{4}} \int_{s_{0}}^{\infty} d s \sigma_{12 \rightarrow 34} s\left(s-s_{0}\right) K_{2}(\sqrt{s} / T),
$$

where $s_{0}$ is the minimum of $s$.

Open Access. This article is distributed under the terms of the Creative Commons Attribution License (CC-BY 4.0), which permits any use, distribution and reproduction in any medium, provided the original author(s) and source are credited.

\section{References}

[1] V.C. Rubin and J. Ford, W.Kent, Rotation of the Andromeda Nebula from a Spectroscopic Survey of Emission Regions, Astrophys. J. 159 (1970) 379 [INSPIRE].

[2] D. Clowe et al., A direct empirical proof of the existence of dark matter, Astrophys. J. Lett. 648 (2006) L109 [astro-ph/0608407] [INSPIRE]. 
[3] Planck collaboration, Planck 2018 results. VI. Cosmological parameters, Astron. Astrophys. 641 (2020) A6 [arXiv: 1807.06209] [INSPIRE].

[4] L. Roszkowski, E.M. Sessolo and S. Trojanowski, WIMP dark matter candidates and searches - current status and future prospects, Rept. Prog. Phys. 81 (2018) 066201 [arXiv: 1707.06277] [INSPIRE].

[5] L.J. Hall, K. Jedamzik, J. March-Russell and S.M. West, Freeze-In Production of FIMP Dark Matter, JHEP 03 (2010) 080 [arXiv:0911.1120] [INSPIRE].

[6] F. Elahi, C. Kolda and J. Unwin, Ultra Violet Freeze-in, JHEP 03 (2015) 048 [arXiv: 1410.6157] [INSPIRE].

[7] N. Bernal, M. Heikinheimo, T. Tenkanen, K. Tuominen and V. Vaskonen, The Dawn of FIMP Dark Matter: A Review of Models and Constraints, Int. J. Mod. Phys. A 32 (2017) 1730023 [arXiv: 1706.07442 ] [INSPIRE].

[8] G. Krnjaic, Freezing In, Heating Up, and Freezing Out: Predictive Nonthermal Dark Matter and Low-Mass Direct Detection, JHEP 10 (2018) 136 [arXiv:1711.11038] [INSPIRE].

[9] J. Berger, D. Croon, S. El Hedri, K. Jedamzik, A. Perko and D.G.E. Walker, Dark matter amnesia in out-of-equilibrium scenarios, JCAP 02 (2019) 051 [arXiv: 1812.08795] [INSPIRE].

[10] K.R. Dienes and B. Thomas, Dynamical Dark Matter: I. Theoretical Overview, Phys. Rev. D 85 (2012) 083523 [arXiv:1106.4546] [INSPIRE].

[11] K.R. Dienes and B. Thomas, Dynamical Dark Matter: II. An Explicit Model, Phys. Rev. D 85 (2012) 083524 [arXiv: 1107.0721] [INSPIRE].

[12] K.R. Dienes, F. Huang, S. Su and B. Thomas, Dynamical Dark Matter from Strongly-Coupled Dark Sectors, Phys. Rev. D 95 (2017) 043526 [arXiv:1610.04112] [INSPIRE].

[13] G. Kane, K. Sinha and S. Watson, Cosmological Moduli and the Post-Inflationary Universe: A Critical Review, Int. J. Mod. Phys. D 24 (2015) 1530022 [arXiv:1502.07746] [inSPIRE].

[14] B.S. Acharya, G. Kane, S. Watson and P. Kumar, A Non-thermal WIMP Miracle, Phys. Rev. D 80 (2009) 083529 [arXiv:0908.2430] [INSPIRE].

[15] A. Berlin, D. Hooper and G. Krnjaic, PeV-Scale Dark Matter as a Thermal Relic of a Decoupled Sector, Phys. Lett. B $\mathbf{7 6 0}$ (2016) 106 [arXiv:1602.08490] [INSPIRE].

[16] A. Berlin, D. Hooper and G. Krnjaic, Thermal Dark Matter From A Highly Decoupled Sector, Phys. Rev. D 94 (2016) 095019 [arXiv: 1609.02555] [InSPIRE].

[17] L. Heurtier and F. Huang, Inflaton portal to a highly decoupled EeV dark matter particle, Phys. Rev. D 100 (2019) 043507 [arXiv: 1905.05191] [InSPIRE].

[18] B. Holdom, Two U(1)'s and Epsilon Charge Shifts, Phys. Lett. B 166 (1986) 196 [inSPIRE].

[19] L.B. Okun, Limits of electrodynamics: paraphotons?, Sov. Phys. JETP 56 (1982) 502 [INSPIRE].

[20] A. Falkowski, J. Juknevich and J. Shelton, Dark Matter Through the Neutrino Portal, arXiv:0908.1790 [INSPIRE].

[21] V. Gonzalez Macias and J. Wudka, Effective theories for Dark Matter interactions and the neutrino portal paradigm, JHEP 07 (2015) 161 [arXiv:1506.03825] [INSPIRE].

[22] P. Ko and Y. Tang, IceCube Events from Heavy DM decays through the Right-handed Neutrino Portal, Phys. Lett. B 751 (2015) 81 [arXiv:1508.02500] [INSPIRE]. 
[23] A. Hamze, C. Kilic, J. Koeller, C. Trendafilova and J.-H. Yu, Lepton-Flavored Asymmetric Dark Matter and Interference in Direct Detection, Phys. Rev. D 91 (2015) 035009 [arXiv: 1410.3030] [INSPIRE].

[24] J.-H. Yu, Hidden Gauged U(1) Model: Unifying Scotogenic Neutrino and Flavor Dark Matter, Phys. Rev. D 93 (2016) 113007 [arXiv:1601.02609] [InSPIRE].

[25] V. González-Macías, J.I. Illana and J. Wudka, A realistic model for Dark Matter interactions in the neutrino portal paradigm, JHEP 05 (2016) 171 [arXiv:1601.05051] [INSPIRE].

[26] M. Escudero, N. Rius and V. Sanz, Sterile neutrino portal to Dark Matter I: The U(1) $B-L$ case, JHEP 02 (2017) 045 [arXiv: 1606.01258] [INSPIRE].

[27] M. Escudero, N. Rius and V. Sanz, Sterile Neutrino portal to Dark Matter II: Exact Dark symmetry, Eur. Phys. J. C 77 (2017) 397 [arXiv: 1607.02373] [INSPIRE].

[28] B. Batell, T. Han and B. Shams Es Haghi, Indirect Detection of Neutrino Portal Dark Matter, Phys. Rev. D 97 (2018) 095020 [arXiv: 1704.08708] [INSPIRE].

[29] B. Batell, T. Han, D. McKeen and B. Shams Es Haghi, Thermal Dark Matter Through the Dirac Neutrino Portal, Phys. Rev. D 97 (2018) 075016 [arXiv:1709.07001] [InSPIRE].

[30] M. Chianese and S.F. King, The Dark Side of the Littlest Seesaw: freeze-in, the two right-handed neutrino portal and leptogenesis-friendly fimpzillas, JCAP 09 (2018) 027 [arXiv: 1806.10606] [INSPIRE].

[31] L. Bian and Y.-L. Tang, Thermally modified sterile neutrino portal dark matter and gravitational waves from phase transition: The Freeze-in case, JHEP 12 (2018) 006 [arXiv: 1810.03172] [INSPIRE].

[32] M. Blennow, E. Fernandez-Martinez, A. Olivares-Del Campo, S. Pascoli, S. Rosauro-Alcaraz and A.V. Titov, Neutrino Portals to Dark Matter, Eur. Phys. J. C 79 (2019) 555 [arXiv: 1903.00006] [INSPIRE].

[33] M. Chianese, B. Fu and S.F. King, Minimal Seesaw extension for Neutrino Mass and Mixing, Leptogenesis and Dark Matter: FIMPzillas through the Right-Handed Neutrino Portal, JCAP 03 (2020) 030 [arXiv:1910.12916] [inSPIRE].

[34] J.F. Cherry, A. Friedland and I.M. Shoemaker, Neutrino Portal Dark Matter: From Dwarf Galaxies to IceCube, arXiv:1411.1071 [INSPIRE].

[35] A. Berlin and N. Blinov, Thermal neutrino portal to sub-MeV dark matter, Phys. Rev. D 99 (2019) 095030 [arXiv:1807.04282] [INSPIRE].

[36] C. Cheung, G. Elor, L.J. Hall and P. Kumar, Origins of Hidden Sector Dark Matter I: Cosmology, JHEP 03 (2011) 042 [arXiv: 1010.0022] [INSPIRE].

[37] C. Cheung, G. Elor, L.J. Hall and P. Kumar, Origins of Hidden Sector Dark Matter II: Collider Physics, JHEP 03 (2011) 085 [arXiv: 1010.0024] [InSPIRE].

[38] X. Chu, T. Hambye and M.H.G. Tytgat, The Four Basic Ways of Creating Dark Matter Through a Portal, JCAP 05 (2012) 034 [arXiv:1112.0493] [INSPIRE].

[39] N. Bernal, X. Chu, C. Garcia-Cely, T. Hambye and B. Zaldivar, Production Regimes for Self-Interacting Dark Matter, JCAP 03 (2016) 018 [arXiv:1510.08063] [INSPIRE].

[40] S. Tulin and H.-B. Yu, Dark Matter Self-interactions and Small Scale Structure, Phys. Rept. 730 (2018) 1 [arXiv: 1705. 02358] [INSPIRE]. 
[41] Y. Chikashige, R.N. Mohapatra and R.D. Peccei, Spontaneously Broken Lepton Number and Cosmological Constraints on the Neutrino Mass Spectrum, Phys. Rev. Lett. 45 (1980) 1926 [INSPIRE].

[42] Y. Chikashige, R.N. Mohapatra and R.D. Peccei, Are There Real Goldstone Bosons Associated with Broken Lepton Number?, Phys. Lett. B 98 (1981) 265 [INSPIRE].

[43] J. Schechter and J.W.F. Valle, Neutrino Decay and Spontaneous Violation of Lepton Number, Phys. Rev. D 25 (1982) 774 [InSPIRE].

[44] G.B. Gelmini and M. Roncadelli, Left-Handed Neutrino Mass Scale and Spontaneously Broken Lepton Number, Phys. Lett. B 99 (1981) 411 [INSPIRE].

[45] G. Gelmini, D.N. Schramm and J.W.F. Valle, Majorons: A Simultaneous Solution to the Large and Small Scale Dark Matter Problems, Phys. Lett. B 146 (1984) 311 [inSPIRE].

[46] M. Lattanzi, S. Riemer-Sorensen, M. Tortola and J.W.F. Valle, Updated CMB and $x$ - and $\gamma$-ray constraints on Majoron dark matter, Phys. Rev. D $8 \mathbf{8}$ (2013) 063528 [arXiv: 1303.4685] [INSPIRE].

[47] V. Berezinsky and J.W.F. Valle, The KeV majoron as a dark matter particle, Phys. Lett. B 318 (1993) 360 [hep-ph/9309214] [INSPIRE].

[48] F. Bazzocchi, M. Lattanzi, S. Riemer-Sørensen and J.W.F. Valle, X-ray photons from late-decaying majoron dark matter, JCAP 08 (2008) 013 [arXiv:0805.2372] [INSPIRE].

[49] P.-H. Gu, E. Ma and U. Sarkar, Pseudo-Majoron as Dark Matter, Phys. Lett. B 690 (2010) 145 [arXiv: 1004.1919] [INSPIRE].

[50] Y. Abe, Y. Hamada, T. Ohata, K. Suzuki and K. Yoshioka, TeV-scale Majorogenesis, JHEP 07 (2020) 105 [arXiv:2004.00599] [INSPIRE].

[51] P.F. de Salas and S. Pastor, Relic neutrino decoupling with flavour oscillations revisited, JCAP 07 (2016) 051 [arXiv:1606.06986] [INSPIRE].

[52] C. Boehm, M.J. Dolan and C. McCabe, Increasing Neff with particles in thermal equilibrium with neutrinos, JCAP 12 (2012) 027 [arXiv: 1207.0497] [INSPIRE].

[53] C.A. Argüelles, A. Diaz, A. Kheirandish, A. Olivares-Del-Campo, I. Safa and A.C. Vincent, Dark Matter Annihilation to Neutrinos, arXiv:1912.09486 [INSPIRE].

[54] Super-Kamiokande collaboration, The Super-Kamiokande detector, Nucl. Instrum. Meth. A 501 (2003) 418 [INSPIRE].

[55] Super-Kamiokande collaboration, Measurements of the atmospheric neutrino flux by Super-Kamiokande: energy spectra, geomagnetic effects, and solar modulation, Phys. Rev. D 94 (2016) 052001 [arXiv: 1510.08127] [INSPIRE].

[56] F. Kahlhoefer, M. Kaplinghat, T.R. Slatyer and C.-L. Wu, Diversity in density profiles of self-interacting dark matter satellite halos, JCAP 12 (2019) 010 [arXiv:1904.10539] [INSPIRE].

[57] E. Papastergis, R. Giovanelli, M.P. Haynes and F. Shankar, Is there a "too big to fail" problem in the field?, Astron. Astrophys. 574 (2015) A113 [arXiv:1407.4665] [INSPIRE].

[58] S. Tulin, H.-B. Yu and K.M. Zurek, Beyond Collisionless Dark Matter: Particle Physics Dynamics for Dark Matter Halo Structure, Phys. Rev. D 87 (2013) 115007 [arXiv: 1302.3898] [INSPIRE]. 
[59] K. Choi, C.W. Kim, J. Kim and W.P. Lam, Constraints on the Majoron Interactions From the Supernova SN1987A, Phys. Rev. D 37 (1988) 3225 [INSPIRE].

[60] Y. Farzan, Bounds on the coupling of the Majoron to light neutrinos from supernova cooling, Phys. Rev. D 67 (2003) 073015 [hep-ph/0211375] [INSPIRE].

[61] L. Heurtier and Y. Zhang, Supernova Constraints on Massive (Pseudo)Scalar Coupling to Neutrinos, JCAP 02 (2017) 042 [arXiv:1609.05882] [INSPIRE].

[62] A. de Gouvêa, P.S.B. Dev, B. Dutta, T. Ghosh, T. Han and Y. Zhang, Leptonic Scalars at the LHC, JHEP 07 (2020) 142 [arXiv:1910.01132] [INSPIRE].

[63] J. König, A. Merle and M. Totzauer, keV Sterile Neutrino Dark Matter from Singlet Scalar Decays: The Most General Case, JCAP 11 (2016) 038 [arXiv: 1609.01289] [InSPIRE].

[64] R. Murgia, A. Merle, M. Viel, M. Totzauer and A. Schneider, "Non-cold" dark matter at small scales: a general approach, JCAP 11 (2017) 046 [arXiv: 1704.07838] [INSPIRE].

[65] K.R. Dienes, F. Huang, J. Kost, S. Su and B. Thomas, Deciphering the archaeological record: Cosmological imprints of nonminimal dark sectors, Phys. Rev. D 101 (2020) 123511 [arXiv: 2001.02193] [INSPIRE].

[66] P. Gondolo and G. Gelmini, Cosmic abundances of stable particles: Improved analysis, Nucl. Phys. B 360 (1991) 145 [InSPIRE]. 